\title{
Humanized Bispecific Antibody (mPEGxHER2) Rapidly Confers PEGylated nanoaparticles Tumor Specificity for Multimodality Imaging in Breast Cancer
}

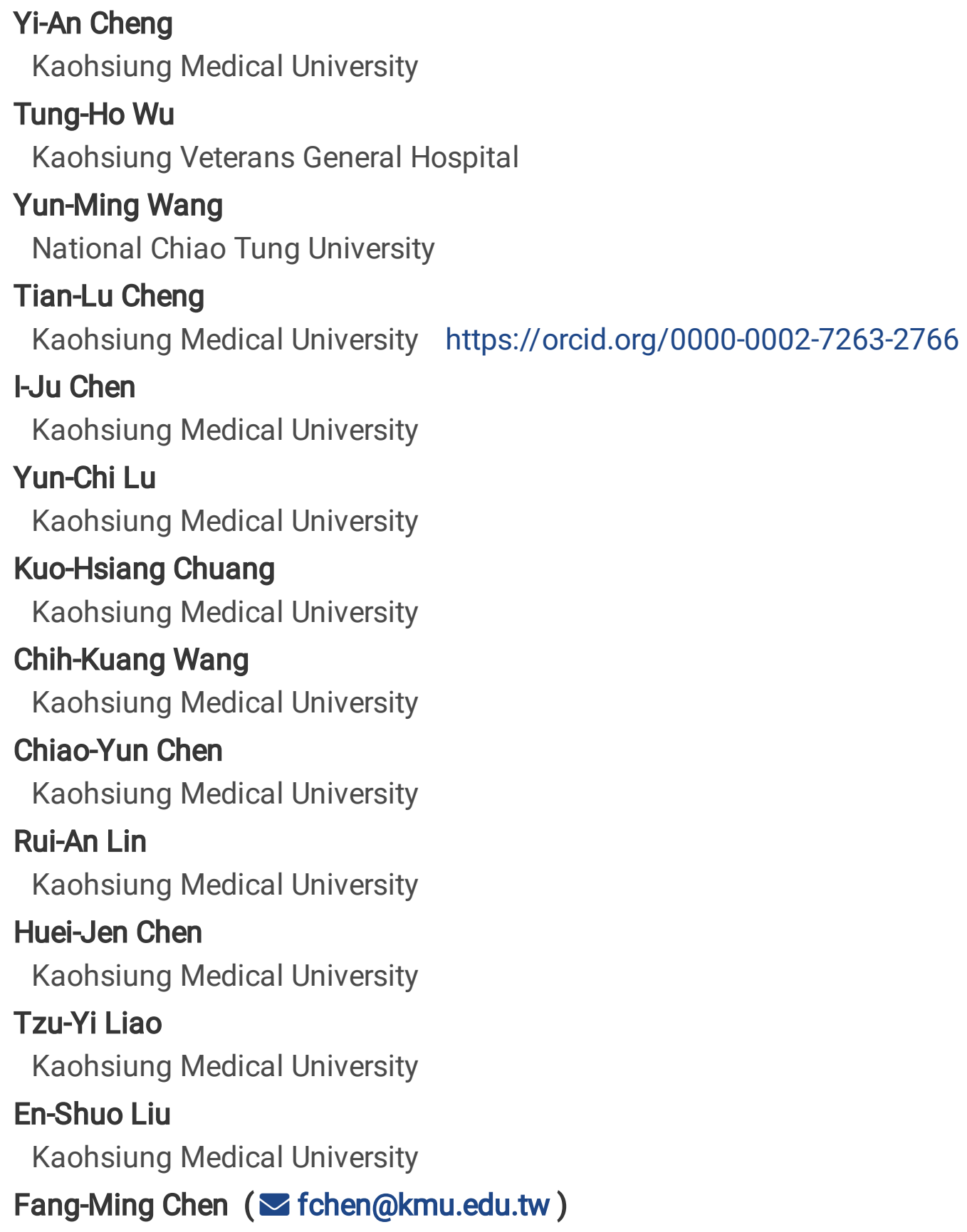


Keywords: Bispecific Antibody, PEGylated nanocparticle, nano-contrast agent, multimodality image, polyethylene glycol, anti-PEG antibody, one-step formulation, Tumor Specificity, targeted image

Posted Date: May 17th, 2020

DOI: https://doi.org/10.21203/rs.3.rs-28604/v1

License: (c) (1) This work is licensed under a Creative Commons Attribution 4.0 International License. Read Full License

Version of Record: A version of this preprint was published on August 27th, 2020. See the published version at https://doi.org/10.1186/s12951-020-00680-9. 


\title{
Humanized Bispecific Antibody (mPEG×HER2) Rapidly Confers PEGylated nanoaparticles Tumor Specificity for Multimodality Imaging in Breast Cancer
}

\author{
$\underline{\text { Yi-An Cheng }}^{1,13, \#, \text { Tung-Ho Wu }}{ }^{2, \#}$, Yun-Ming Wang ${ }^{3}$, Tian-Lu Cheng ${ }^{1,4,5,12}$, I-Ju Chen ${ }^{1}$, Yun-Chi \\ Lu $^{1,12}$, Kuo-Hsiang Chuang ${ }^{6}$, Chih-Kuang Wang ${ }^{13}$, Chiao-Yun, Chen ${ }^{7,8}$, Rui-An, Lin ${ }^{4}$, Chen-Huei \\ $\mathrm{Jen}^{4}$, Tzu-Yi, Liao ${ }^{4}$, En-Shuo, Liu ${ }^{1}, \underline{\text { Fang-Ming Chen }}{ }^{9-12^{*}}$
}

${ }^{1}$ Department of Biomedical Science and Environmental Biology, Kaohsiung Medical University, No.100, Shin-Chuan 1st Road, Sanmin Dist., Kaohsiung, Taiwan

${ }^{2}$ Cardiovascular division of surgical department, Kaohsiung Veterans General Hospital, No.386, Dazhong 1st Rd., Zuoying Dist., Kaohsiung, Taiwan

${ }^{3}$ Department of Biological Science and Technology, Institute of Molecular Medicine and Bioengineering, National Chiao Tung University, No. 1001, University Road, Hsinchu, Taiwan

${ }^{4}$ Graduate Institute of Medicine, Kaohsiung Medical University, No.100, Shin-Chuan 1st Road, Sanmin Dist., Kaohsiung, Taiwan

${ }^{5}$ Department of Medical Research, Kaohsiung Medical University Hospital, No. 100, Tzyou 1st Road, Kaohsiung, Taiwan

${ }^{6}$ Graduate Institute of Pharmacognosy, Taipei Medical University, No.172-1, Sec. 2, Keelung Rd., Taipei, Taiwan

${ }^{7}$ Department of Radiology, Faculty of Medicine, College of Medicine, Kaohsiung Medical University, No.100, ShinChuan 1st Road, Sanmin Dist., Kaohsiung, Taiwan

${ }^{8}$ Department of Medical Imaging, Kaohsiung Medical University Hospital, No. 100, Tzyou 1st Road, Kaohsiung, Taiwan

${ }^{9}$ Division of Breast Surgery, Department of Surgery, Kaohsiung Medical University Hospital, No. 100, Tzyou 1st Road, Kaohsiung, Taiwan

${ }^{10}$ Department of Surgery, Kaohsiung Municipal Ta-Tung Hospital, No. 100, Tzyou 1st Road, Kaohsiung, Taiwan

${ }^{11}$ Department of Surgery, Faculty of Medicine, College of Medicine, Kaohsiung Medical University, No. 100, Tzyou 1st Road, Kaohsiung, Taiwan

${ }^{12}$ Drug Development and Value Creation Research Center, Kaohsiung Medical University, No.100, Shin-Chuan 1st Road, Sanmin Dist., Kaohsiung, Taiwan

${ }^{13}$ Department of Medicinal and Applied Chemistry, Kaohsiung Medical University, No.100, Shin-Chuan 1st Road, Sanmin Dist., Kaohsiung, Taiwan

\# These authors contributed equally to this work.

${ }^{*}$ Corresponding author: Department of Surgery, Faculty of Medicine, College of Medicine, Kaohsiung Medical University, 100 Shin-Chuan 1st Rd. Kaohsiung City 807 Taiwan. Tel.: +886 7 3121101. Fax: +886 7 321-2062. E-mail: fchen@kmu.edu.tw 


\section{Abstract \\ Background:}

Developing a universal strategy to improve the specificity and sensitivity of PEGylated nanoaparticles (PEG-NPs) for assisting in the diagnosis of tumors is important in multimodality imaging. Here, we developed the anti-methoxypolyethylene glycol (mPEG) bispecific antibody (BsAb; mPEG $\times$ HER2), which has dual specificity for mPEG and human epidermal growth factor receptor 2 (HER2), with a diverse array of PEG-NPs to confer nanoparticles with HER2 specificity and stronger intensity.

\section{Result:}

We used a one-step formulation to rapidly modify the nanoprobes with $\mathrm{mPEG} \times \mathrm{HER} 2$ and optimized the modified ratio of BsAbs on several PEG-NPs (Lipo-DiR, SPIO, Qdot and AuNP). The $\alpha$ HER2/PEG-NPs could specifically target MCF7/HER2 cells $\left(\right.$ HER2 $^{+}$) but not MCF7/neo1 cells (HER2 ${ }^{-}$). The $\alpha$ HER2/Lipo-DiR and $\alpha$ HER2/SPIO could enhance the sensitivity of untargeted PEGNPs on MCF7/HER2 (HER2 ${ }^{+}$). In in vivo imaging, $\alpha$ HER2/Lipo-DiR and $\alpha$ HER2/SPIO increased the specific targeting and enhanced mPEG-nanoprobe accumulation 161\% and 187\%, respectively, in HER2-overexpressing tumors.

\section{Conclusion:}

mPEG $\times$ HER2, therefore, provided a simple one-step formulation to confer HER2-specific targeting and enhanced sensitivity and contrast intensity on HER2 positive tumors for multimodality imaging.

Keywords: Bispecific Antibody, PEGylated nanocparticle, nano-contrast agent, multimodality image, polyethylene glycol, anti-PEG antibody, one-step formulation, Tumor Specificity, targeted image 


\section{Introduction}

Non-invasive imaging for in vivo tracking of the location and size of tumors is very important in cancer therapy and diagnostics. Optical imaging (OI) is relatively inexpensive and robust for all kinds of molecular and cellular processes in small animals, but clinical applications are hindered by limited depth penetration[1]. Magnetic resonance imaging (MRI) has spectacular resolution and is particularly well suited for examining non-bony parts and soft tumors (e.g. breast, brain, etc.) in the clinic, but imaging sensitivity is inferior to nuclear techniques[2]. Nuclear imaging is characterized by high sensitivity, but suffers from poor spatial and temporal resolution[3]. Thus, development of multimodality imaging protocols can help overcome the limitations of single imaging modalities[4]. Many nano-contrast agents have been developed such as liposomes, microbubbles, superparamagnetic iron oxide (SPIO), AuNP and so on[5]. Most contrast agents are modified with methoxy-polyethylene glycol (mPEG) as PEG-NPs (PEG-NPs) which can enhance the biocompatibility and half-life of nanoparticles. However, the water-solubility of mPEG reduces the cell uptake of nanoparticles, thus, the PEG-NPs were reported to just passively accumulate in tumor site via the enhanced permeability and retention (EPR) effect that did not increase the cell uptake of nanoparticles in tumor cells[6], thereby limiting the sensitivity and signal intensity of PEG-NPs[7]. Therefore, active tumor-targeting and cell uptake of PEG-NPs is important to enhance the sensitivity for targeted diagnostics[8].

In order to provide tumor specificity to the PEG-NPs, the anti-tumor antibodies, ligands and peptides were conjugated with nanoprobes to form targeted contrast agents[9-12]. Freedman et al. showed that chemical conjugation of liposomal gadopentetate dimeglumine with anti-transferrin receptor $\mathrm{scFv}$ could increase the pixel intensity of small lung cancers $(100 \mathrm{~mm})$ in MRI images compared untargeted liposomes[13]. Chemical conjugation of anti-HER2/EGFR bispecific antibody to SPIO significantly enhanced the relative contrast enhancements in SKBR-3 tumors (HER2 ${ }^{+}$) as compared to colo-205 tumors (HER2') at $24 \mathrm{~h}$ post-injection[14]. However, the chemical conjugation of the functional groups of antibodies to PEG-NPs caused antibody dysfunction, because the coupling site blocks the antigen-binding site of antibody and chemical reagents alter the protein structure. Protein adaptors, such as protein $\mathrm{G}$, biotin and streptavidin, have been developed to non-covalently modify nanoparticles for stable the structure of antibody. For example, streptavidin was used as an adaptor to connect the biotinylated anti-CD45RO antibody and biotinylated PEGylated lipid nanoparticles for selective targeting into memory $\mathrm{T}$ cells[15]. Protein $\mathrm{G}$ (IgG-binding b2 domain) was conjugated to gold nanoparticles with anti-HER2 antibody for specific targeting to HER2 overexpressing breast cancer[16]. Nevertheless, using exogenous adaptors, which induce 
immunogenicity, is not allowed in the human body, leading to reduce the half-life of PEG-NPs and limiting the rapid development of molecular imaging in clinic. Thus, developing a modification method which is simple, convenient and has low immunogenicity for universal contrast materials is important to improve the tumor specificity and sensitivity of targeted PEG-NPs.

We previously established humanized bispecific antibody (BsAb; $\mathrm{mPEG} \times \mathrm{HER} 2)$ which can bind to the terminal methoxy groups present on PEG chains surrounding PEGylated drugs to confer HER2binding specificity to nanoparticles. Humanized BsAbs can provide non-covalent modification as a simple one-step formulation on PEG-NPs[17]. In this study, we investigated whether multiple PEGNPs (liposome, SPIO, Qdot and AuNP) could be modified by mPEG $\times$ HER2. Additionally, we examined the specific targeting and sensitivity of HER2-targeted nanoparticles in HER2 positive cancer cells using non-invasive imaging. For in vivo imaging, the signal intensity of HER2-targeted Lipo-DiR and SPIO were analyzed on HER2 positive tumors and HER2 negative tumors. This onestep formulation of PEG-NPs with mPEG $\times$ HER2 is a simple method to confer HER2-specific targeting and enhanced sensitivity and contrast intensity on HER2 positive tumors for multimodality diagnostic imaging. (Figure 1)

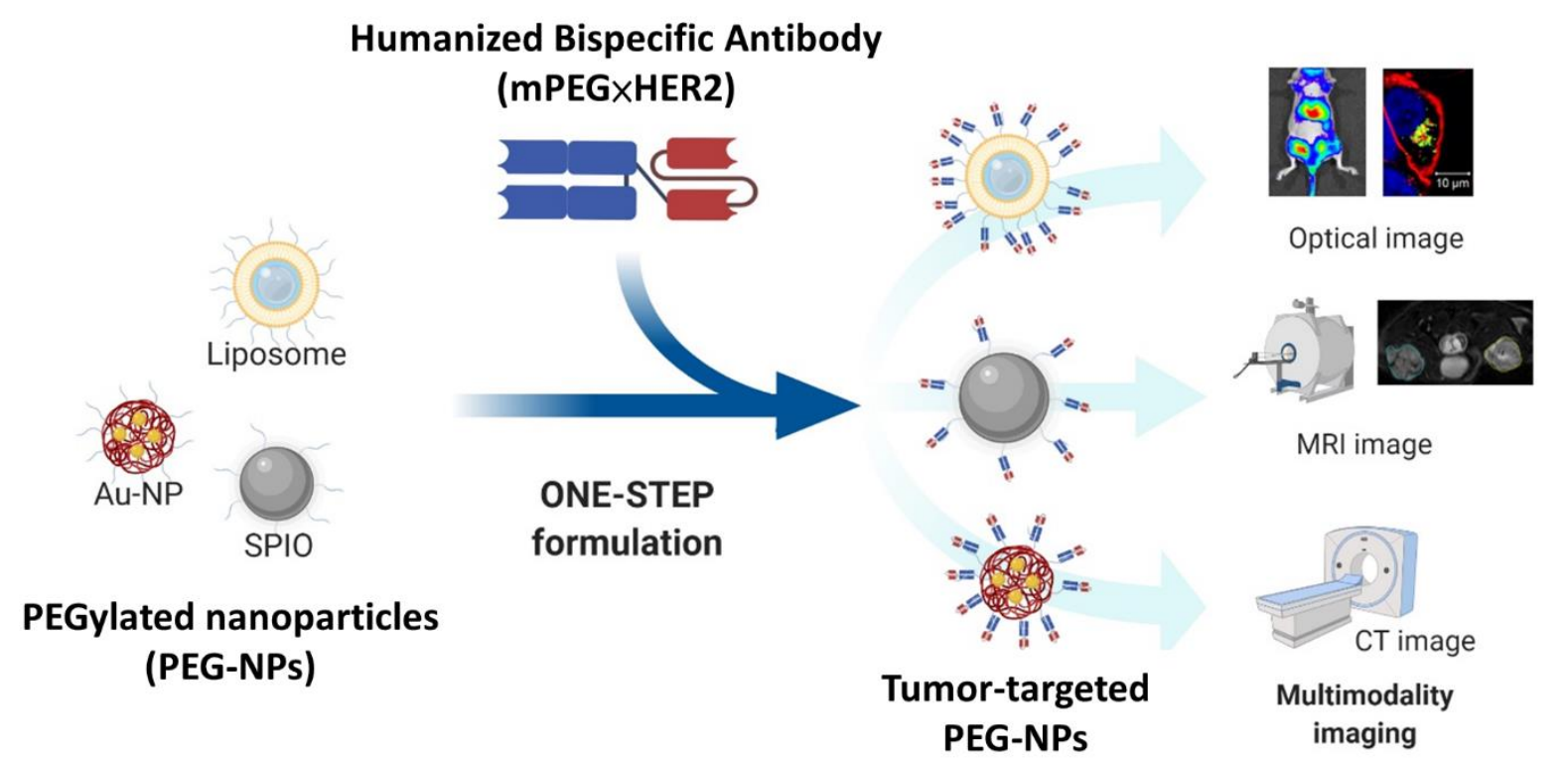

Figure 1. Humanized Bispecific Antibody (mPEG×HER2) Rapidly Confers PEG-NPs Tumor Specificity for Multimodality Imaging in Breast Cancer

anti-mPEG BsAbs (mPEG×markers) provide an easy, universal and one-step formulation for any naive PEG-NPs to accelerate the development of targeted PEG-NPs for multimodality imaging in the clinic. 


\section{Results:}

\section{One-step formulation of PEGylated nanoparticles with mPEG $\times$ HER2}

To demonstrate whether the bispecific antibody (BsAb) could modify the PEG-NPs, we used $\mathrm{mPEG} \times$ HER2 which was constructed from humanized bispecific antibodies by fusing the anti-mPEG Fab and anti-HER2 scFv via peptide linker $(\mathrm{G} 4 \mathrm{~S})_{3}$, and the $\mathrm{mPEG} \times \mathrm{DNS}$ as a negative control which was created by exchanging the anti-HER2 scFv with an anti-DNS scFv, which binds the small chemical hapten dansyl. Frist, we quantified the mPEG concentration on PEG-NPs (Lipo-DiR, SPIO, Qdot and AuNP) by mPEG antibody-based sandwich ELISA ${ }^{136}$, which uses the anti-mPEG backbone antibody as a capture antibody and the $15-2 \mathrm{~b}$ antibody as a detection antibody. And then, different amounts of mPEG $\times$ HER2 were mixed with Lipo-DiR, SPIO, Qdot and AuNP, respectively, and the BsAb to mPEG modification ratio (BsAb:mPEG; mol:mol) was 64:360 to 1:360. The $\alpha$ HER2/PEG-NPs were quantified to the unconjugated BsAb to determine the BsAb conjugation rate of PEG-NPs. The $\alpha$ HER2/PEG-NPs were added to the mPEG-coated ELISA plates and then sequentially treated with anti-human Fab-HRP antibody and ABTS substrate to detect the unconjugated BsAbs. The BsAb conjugation ratio of $\alpha$ HER2/PEG-NPs was calculated by total number of BsAbs minus number of unconjugated BsAbs then divided by total number of BsAbs. As shown in Table 1, the conjugation ratio of $\alpha$ HER2/PEG-NPs (Lipo-DiR, SPIO, Qdot and AuNP) was above 84.7 to 99\% whereas Lipo$\mathrm{DiR}$ and AuNP were observed in the precipitate in the highest BsAb:mPEG ratio group at $24 \mathrm{~h}$ incubation at $4^{\circ} \mathrm{C}$. We further compared the binding ability of the different BsAb:mPEG ratios of $\alpha$ HER2/PEG-NPs (Lipo-DiR, SPIO, Qdot and AuNP) to MCF7/HER2 cells. The $\alpha$ HER2-nanocontrast agents and $\alpha$ DNS-nanoprobes (Lipo-DiR, SPIO, Qdot and AuNP) were incubated with MCF7/HER2 $\left(\mathrm{HER}^{+}\right)$cells, and then the bound PEG-NPs were detected by using the anti-PEG backbone antibody. We choose the optimized BsAb:mPEG ratio of PEG-NPs, which had higher binding efficiency to HER2, of BsAb on Lipo-DiR, SPIO, Qdot and AuNP was 4:360, 64:360, 4:360 and 8:360, respectively (Figure 2). The result indicated that the BsAb could modify the diverse PEG-NPs by a simple onestep method. 
Table 1. The BsAb-conjugation rate of $\alpha$ HER2/PEG-NPs

\begin{tabular}{cccccccc} 
& \multicolumn{7}{c}{ BsAb:mPEG molar ratio } \\
\cline { 2 - 7 } mPEG-probe & $\mathbf{6 4 : 3 6 0}$ & $\mathbf{3 2 : 3 6 0}$ & $\mathbf{1 6 : 3 6 0}$ & $\mathbf{8 : 3 6 0}$ & $\mathbf{4 : 3 6 0}$ & $\mathbf{2 : 3 6 0}$ & $\mathbf{1 : 3 6 0}$ \\
\hline Lipo-DiR & $\mathrm{P}$ & $\mathrm{P}$ & $\mathrm{P}$ & $94.6 \%, \mathrm{P}$ & $94.9 \%$ & $>99 \%^{*}$ & $>99 \%^{*}$ \\
\hline SPIO & $98.6 \%$ & $98 \%$ & $>99 \% *$ & $>99 \% *$ & $\mathrm{~N}$ & $\mathrm{~N}$ & $\mathrm{~N}$ \\
\hline Qdot & $\mathrm{N}$ & $\mathrm{N}$ & $\mathrm{N}$ & $\mathrm{N}$ & $90 \%$ & $91.2 \%$ & $>99 \% *$ \\
\hline Au-NP & $\mathrm{N}$ & $90.8 \%, \mathrm{P}$ & $84.7 \%$ & $88.9 \%$ & $\mathrm{~N}$ & $\mathrm{~N}$ & $\mathrm{~N}$ \\
\hline
\end{tabular}

$\mathrm{P}$ : Observing the precipitate within 1 hour at $4^{\circ} \mathrm{C}$

$\mathrm{N}$ : Not result

*: Undetectable of free BsAb

(A)

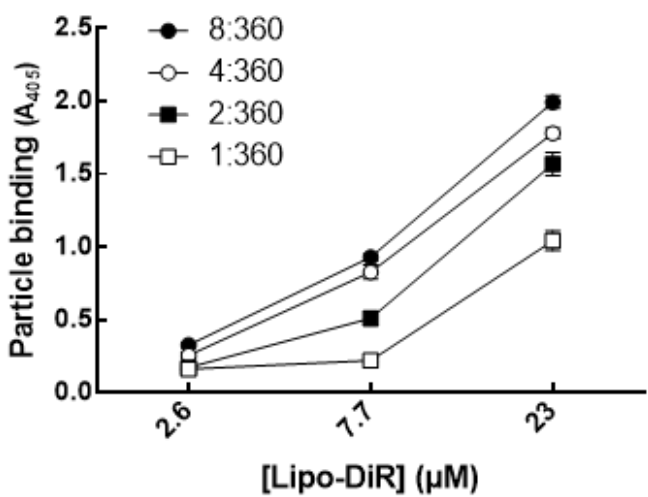

(C)

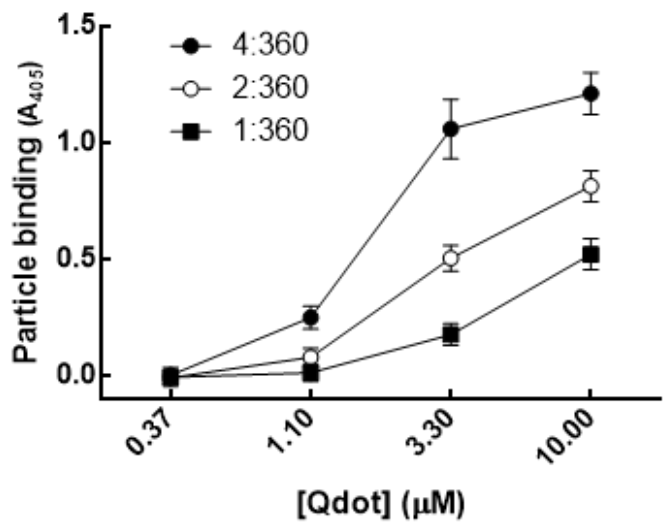

(B)

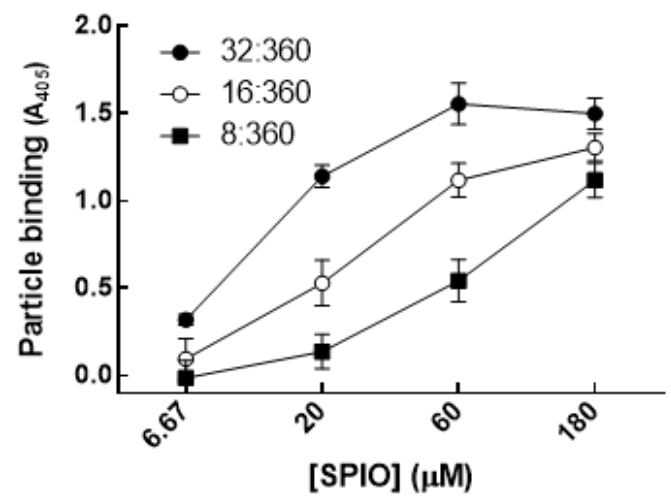

(D)

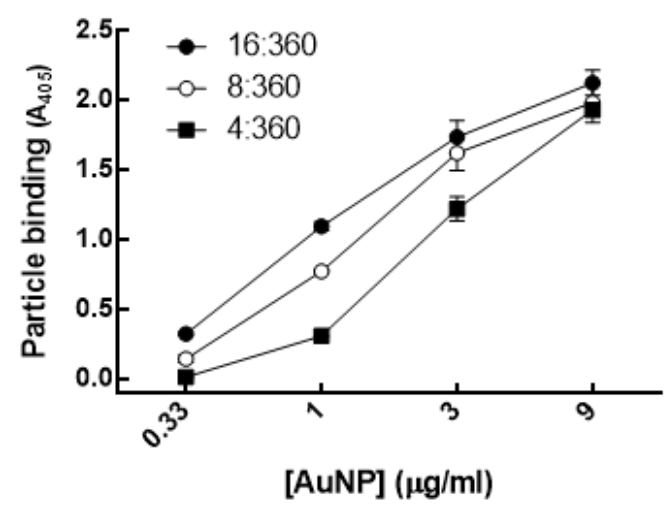

Figure 2. The HER2 binding ability of $\alpha H E R 2 / P E G-N P s$ 
Different BsAb:mPEG ratios of (A) $\alpha$ HER2/Lipo-DiR, (B) $\alpha \mathrm{HER} 2 / \mathrm{SPIO}$, (C) $\alpha \mathrm{HER} 2 / \mathrm{Qdot}$ and (D) $\alpha$ HER2/AuNP were incubated with MCF7/HER2, and then anti-PEG antibody was added to detect PEG-NPs via ELISA ( $n=3$, triplicate). Bars, SD.

\section{The specificity of $\alpha H E R 2 / P E G-N P s$ on HER2-overexpressing cells}

To examine the tumor specificity of optimized targeted PEG-NPs, we used MCF7/HER2 cells which express high levels of HER2, and MCF7/neo1 cells with low levels of HER2. The aHER2nanoprobes and $\alpha \mathrm{DNS}$-nanoprobes (Lipo-DiR, SPIO, Qdot and AuNP) were incubated with MCF7/HER2 (HER2 ${ }^{+}$) cells or MCF7/neo1 (HER2-) cells, and then the mPEGs of PEG-NPs were detected by using the anti-PEG backbone antibody, anti-mouse IgG Fc-HRP and ABTS. As shown in Figure 3, the absorbance value of $\alpha$ HER2/PEG-NPs (Lipo-DiR, SPIO, Qdot and AuNP) was gradually increased as compared with $\alpha$ DNS/PEG-NPs. We demonstrated that mPEG $\times$ HER2 could confer HER2 specificity to the diverse PEG-NPs by one-step formulation.

(A)

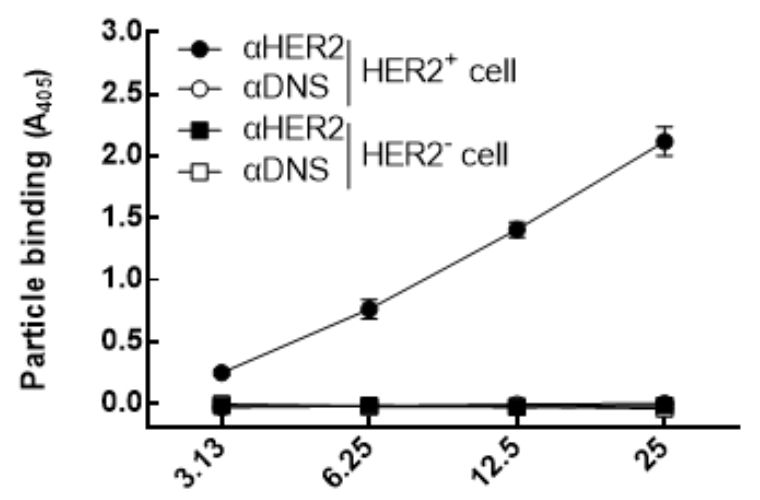

(C)

[Lipo-DiR] ( $\mu \mathrm{M})$

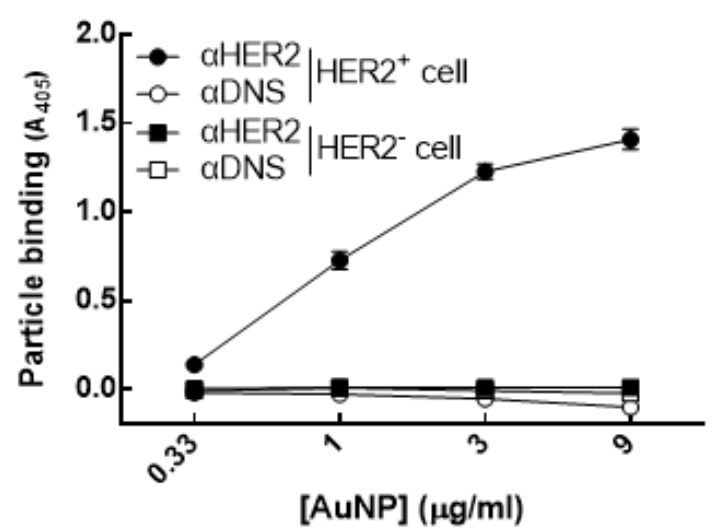

(B)

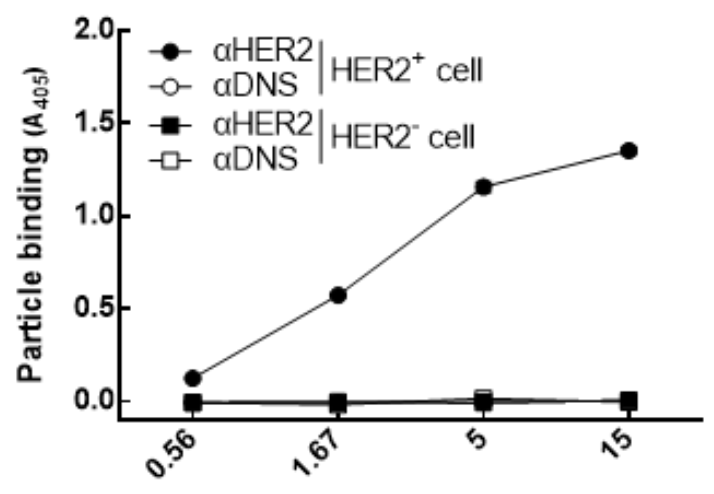

(D)

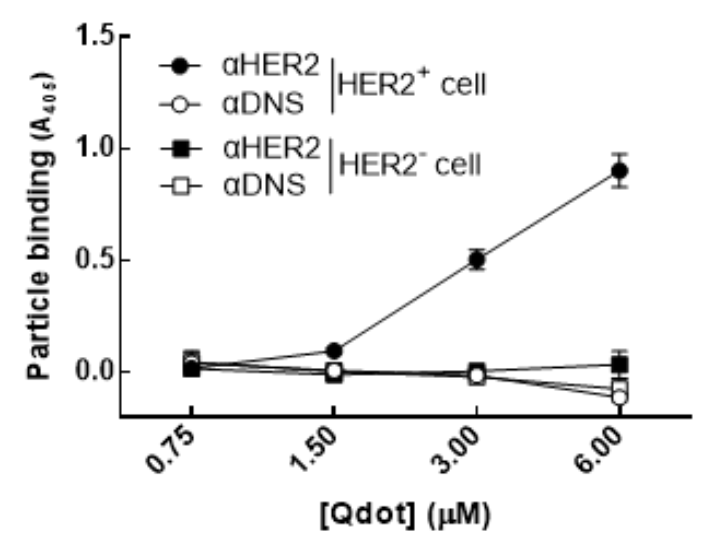

Figure 3. Specificity of $\alpha$ HER2/PEG-NPs for HER2+ cancer cells 
MCF7/HER2 (HER ${ }^{+}$) (circle shape) and MCF7/neo1(HER2-) (square shape) cancer cells in 96-well plates were incubated with $\mathrm{mPEG} \times \mathrm{HER} 2$ (solid shape) and $\mathrm{mPEG} \times \mathrm{DNS}$ (hollow shape) modified with different contrast agents. After washing, bound contrast agents were detected by ELISA ( $\mathrm{n}=3$, triplicate). Bars, SD.

\section{The sensitivity of $\alpha$ HER2/PEG-NPs on HER2-expressing cells}

We wanted to know whether the one-step formulation of PEG-NPs with mPEG $\times$ HER2 improves their sensitivity to HER2 $2^{+}$tumor cells. For optical imaging, we used lipo-DiR as the near-infrared fluorescence liposome mixed with BsAbs. The various concentrations of $\alpha H E R 2 / L i p o-D i R$, $\alpha \mathrm{DNS} /$ Lipo-DiR, or Lipo-DiR were incubated with MCF7/HER2, and then the fluorescence of DiR was detected by IVIS imaging. We calculated the relative fluorescence intensity by dividing the ROI of Lipo-DiR to the cells alone. As shown in Figure 4A, the fluorescence intensity of $\alpha$ HER2/LipoDiR is significantly stronger than that of $\alpha \mathrm{DNS} /$ Lipo-DiR, or Lipo-DiR. At high concentrations, the fluorescence intensity of $\alpha$ HER2/Lipo-DiR was 1.6 fold higher than Lipo-DiR. At low concentrations, $\alpha$ HER2/Lipo-DiR was still 1.2 fold higher than Lipo-DiR (Figure 4B). For MR imaging, $\alpha H E R 2 / S P I O$, $\alpha \mathrm{DNS} / \mathrm{SPIO}$ or SPIO was incubated with MCF7/HER2 and the SPIO accumulation was examined by MRI. As shown in Figure 4C, the strong MR signals as visualized by a darker color were only observed for $\alpha$ HER2/SPIO. After calculation, $\alpha$ HER2/SPIO still had 1.75 fold higher signal intensity (SI) at low concentration $(1.5 \mu \mathrm{g} / \mathrm{ml})$ whereas the signal of $\alpha \mathrm{DNS} / \mathrm{SPIO}$ and SPIO was undetectable. The results showed that the one-step formulation of $\mathrm{mPEG} \times$ HER 2 could enhance the image intensity of the PEGNPs. Additionally, the detectable concentration of the $\alpha$ HER2/PEG-NPs is significantly lower than that of the untargeted PEG-NPs. 

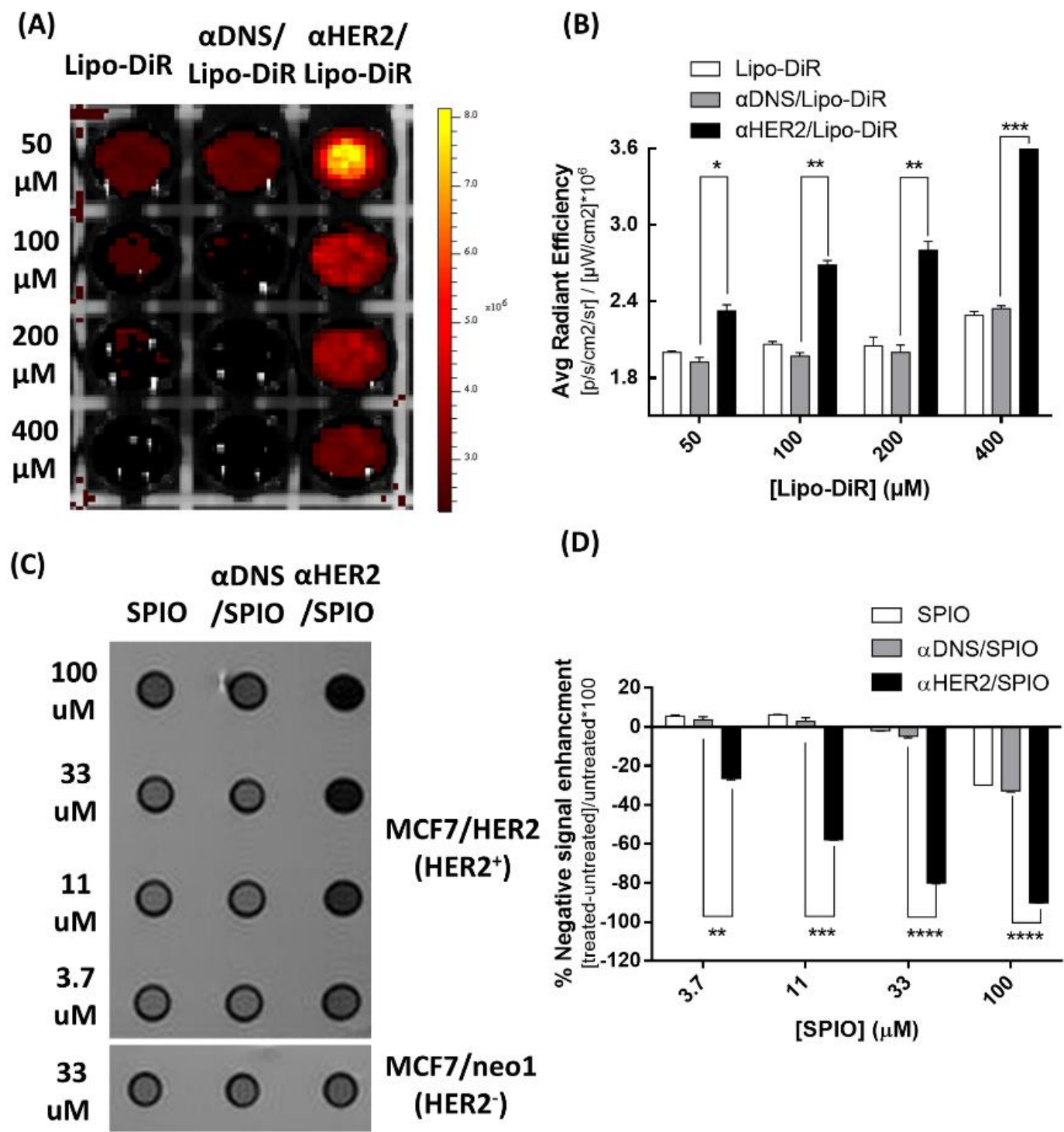

(D)

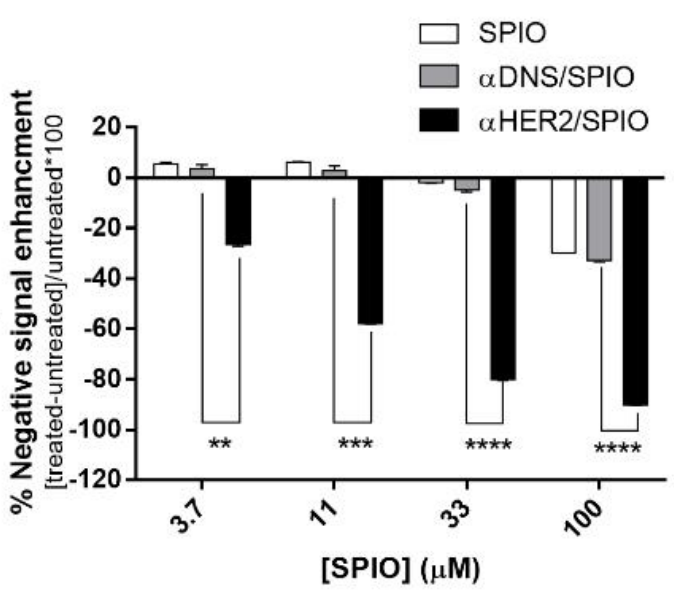

Figure 4. In vitro sensitivity image of $\alpha$ HER2/PEG-NPs

MCF7/HER2 (HER+) cancer cells incubated with HER2 targeted-contrast agent serial dilution concentration. (A) $\alpha H E R 2 / L i p o-D i R, \alpha D N S / L i p o-D i R$ and Lipo-DiR was added to cells. Fluorescence images were obtained by the IVIS spectrum system. (B) The result was the calculation of average radiant efficiency of (A). (C) $\alpha \mathrm{HER} 2 / \mathrm{SPIO}, \alpha \mathrm{DNS} / \mathrm{SPIO}$ and SPIO was added to cells. MR imaging was performed with a 7.0 T MR imaging scanner. (D) The result from (C) was calculated by [treated SI-untreated SI]/ untreated SI*100. $* \mathrm{P}<0.05, * * \mathrm{P}<0.01, * * * \mathrm{P}<0.001, * * * * \mathrm{P}<0.0001$ (unpaired $\mathrm{t}$ test).

\section{The tumor delivery of $\alpha$ HER2/Lipo-DiR on HER2-expressing tumors}

To investigate whether $\mathrm{mPEG} \times$ HER2 can enhance tumor accumulation of PEGylated liposomal DiR (Lipo-DiR) in HER2 over-expressing tumors, we first mixed mPEG $\times$ HER2 with Lipo-DiR to form 
aHER2/Lipo-DiR. Mice bearing MCF7/HER2 (HER2 ${ }^{+}$, in left flank) and MCF7/neo1 (HER2-, in right flank) tumors were intravenously injected with $\alpha$ HER2/Lipo-DiR and Lipo-DiR, and then the fluorescence of DiR was detected by IVIS imaging at $24 \mathrm{~h}, 48 \mathrm{~h}$ and $72 \mathrm{~h}$. The fluorescence signal of $\alpha$ HER2/Lipo-DiR was enhanced in MCF7/HER2 (HER2 ${ }^{+}$) tumors as compared to MCF7/neo1 (HER2-) tumors from $24 \mathrm{~h}$ to $72 \mathrm{~h}$ after probe injection (Fig. 5A). The fluorescence intensity of $\alpha$ HER2/LipoDiR in MCF7/HER2 (HER2 ${ }^{+}$) tumors was 1.61-fold higher than in MCF7/neo1 (HER2-) tumors whereas Lipo-DiR produced 1.06-fold higher fluorescence, respectively (Fig. 5B). The results indicate that $\mathrm{mPEG} \times \mathrm{HER} 2$ can enhance specific targeting of PEG-NPs in HER2-overexpressing tumors.

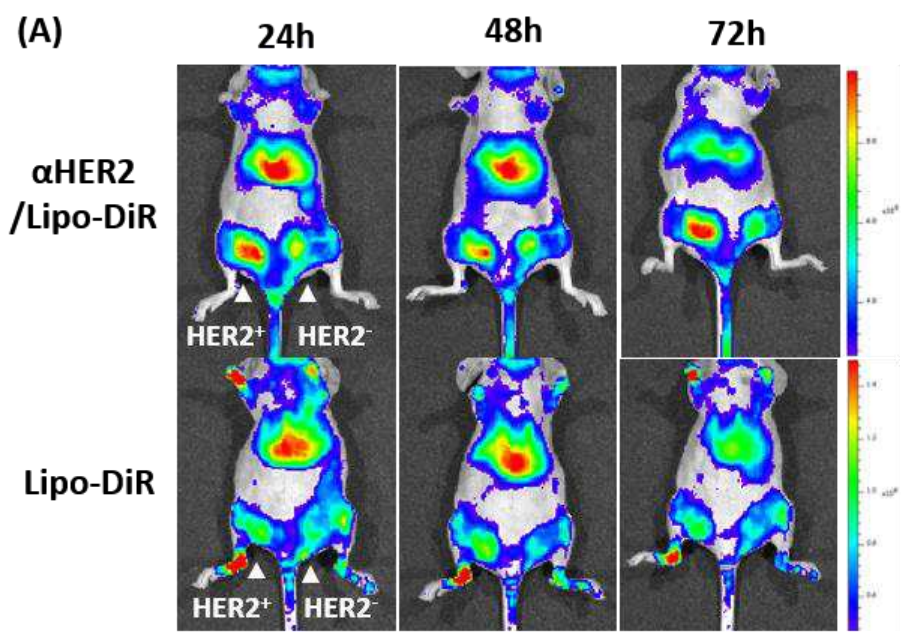

(B)

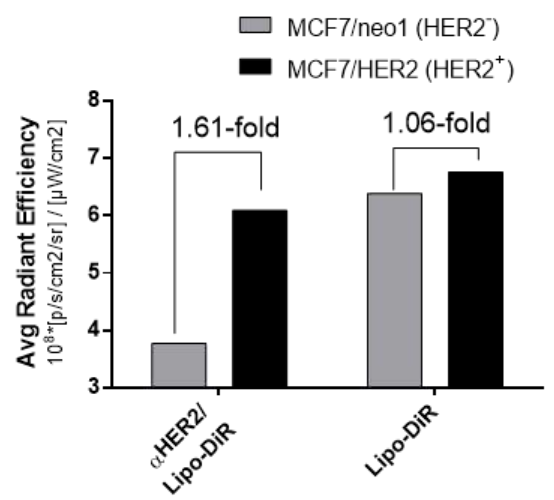

\section{Figure 5. In vivo IVIS imaging of $\alpha H E R 2 / S P I O$ and $\alpha D N S / S P I O$}

The tumor delivery of $\alpha$ HER2/PLD in HER2-overexpressing tumors. (A) $\alpha$ HER2/Lipo-DiR and LipoDiR were intravenously injected in mice bearing HER2high (left flank) and HER2low (right flank) tumors. The fluorescence intensity of DiR was detected at $24 \mathrm{~h}$ and $72 \mathrm{~h}$ after injection by IVIS. (B) Quantification of average radiant efficiency in HER2 $2^{+}$tumor and HER2- tumor at $72 \mathrm{~h}$.

\section{Tumor accumulation and contrast intensity of $\alpha$ HER2/SPIO on HER2-expressing tumors}

To examine the in vivo tumor accumulation of $\alpha$ HER2/SPIO in HER2-positive tumors by MR imaging, $\alpha$ HER2/SPIO or $\alpha$ DNS/SPIO were intravenous injected into mice bearing subcutaneous MCF7/HER2(HER2 ${ }^{+}$) tumors and MCF7/HER2(HER2 ${ }^{-}$). The mean signal intensity (SI) was detected by T2-weighted fast spin-echo sequence $7.0 \mathrm{~T}$ imaging for every $3 \mathrm{~mm}$ sectioning thickness at $0 \mathrm{~h}$ and $24 \mathrm{~h}$ post-injection time. The percentage of negative contrast enhancement was calculated as the $\mathrm{SI}_{24}$ minus the $\mathrm{SI}_{0}$ divided by the $\mathrm{SI}_{0}$. The negative contrast enhancements of $\alpha \mathrm{HER} 2 / \mathrm{SPIO}$ were $-32.8 \%$ (MCF7/HER2) and -17.5\% (MCF7/neo1) whereas those of $\alpha$ DNS/SPIO were -23.8\% (MCF7/HER2) and $-23.3 \%$ (MCF7/neo1) (Figure 6A). We calculated the relative contrast enhancements in 
MCF7/HER2 tumors as compared to MCF7/neo1. The relative contrast enhancements of $\alpha$ HER2/SPIO were $187 \%$, higher than the $102 \%$ of $\alpha \mathrm{DNS} / \mathrm{SPIO}$ (Figure 6B). The results indicate that $\mathrm{mPEG} \times \mathrm{HER} 2$ can enhance specific targeting and accumulation of PEG-NPs in HER2-overexpressing tumors.
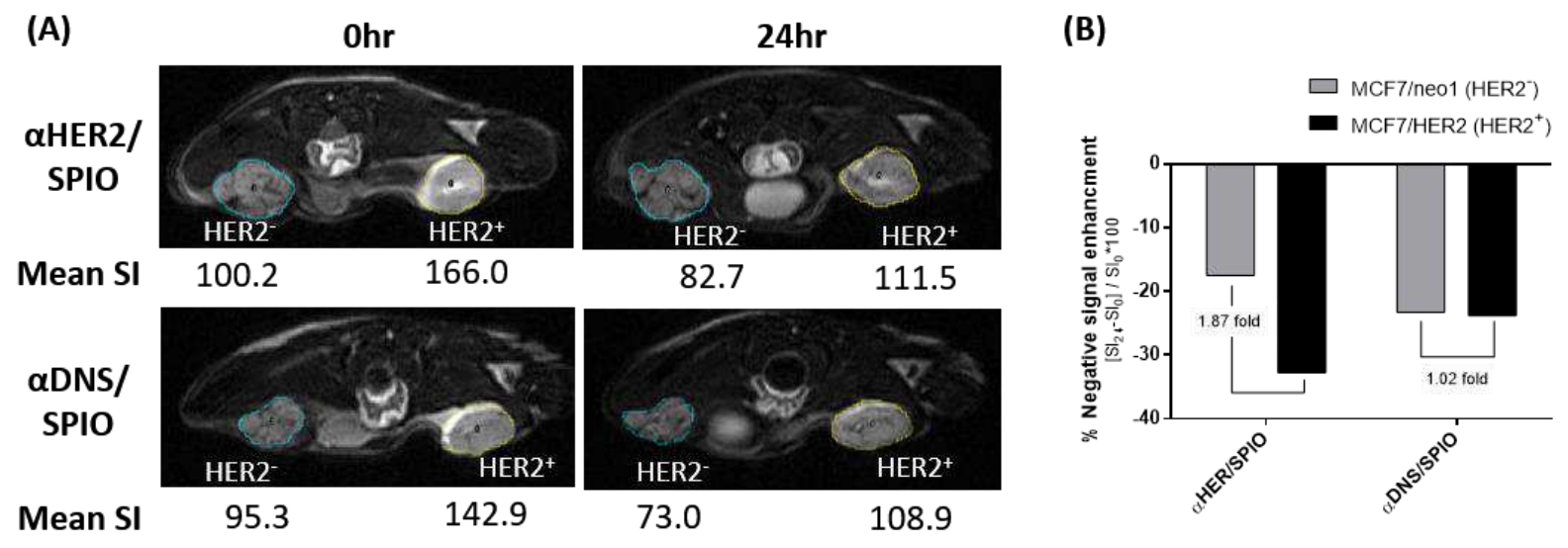

Figure 6. In vivo MR imaging of $\alpha H E R 2 / S P I O$ and $\alpha D N S / S P I O$

Nude mice bearing MCF7/HER2 (right m.f.p) and MCF7/neo1 (left m.f.p) tumors were intravenously injected with $\alpha H E R 2 / S P I O$ and $\alpha$ DNS/ SPIO (10 mg/kg). (A) Mice were sequentially imaged at pretreatment and $24 \mathrm{~h}$ with a 7.0 T MR imaging scanner. (B) The result was calculated as the percentage of negative signal enhancement by [pretreated signal intensity $\left(\mathrm{SI}_{0}\right)$-treated signal intensity $\left.\left(\mathrm{SI}_{24}\right)\right] / \mathrm{SI}_{0} * 100$. 


\section{Discussion}

We have successfully demonstrated that anti-mPEG BsAbs (mPEG $\times$ HER2) could be conjugated to PEG-NPs by a one-step process to confer HER2 specificity on MCF7/HER2 (HER2-expressing cells) for multimodality imaging. Without changing the structure of nanoparticles, the PEG-NPs (liposome, Qdot, AuNP and SPIO) were enhanced with HER2 targeting by modification at an optimized mPEG $\times$ HER2-conjugation ratio. In non-invasive imaging, $\alpha H E R 2 / L i p o-D i R$ and $\alpha$ HER2/SPIO could enhance the intensity of the image signal on MCF7/HER2 tumors in MRI imaging, as compared with untargeted PEG-NPs. Due to the one-step modification, the anti-mPEG BsAb did not change the structure of the nanoparticles. This strategy can be easily applied to a diverse array of PEG-NPs for multimodality imaging. Moreover, the tumor specificity of anti-mPEG BsAbs can also be changed for different markers for corresponding diseases. The anti-mPEG BsAbs can confer tumor targeting to a variety of PEG-NPs. This technique can accelerate the development of targeted PEGNPs to give more accurate diagnoses.

Developing a universal strategy to confer tumor specificity to each mPEG-nanoprobe is important for multimodality imaging. Thus, many studies have focused on developing bifunctional proteins as a universal antibody-conjugation method suitable for any PEG-NPs. For example, Hussain, et al. generated the EGFR $\times$ SNAP bifunctional protein by fusing anti-EGFR scFv and SNAP tag, which allowed covalent attachment of $\mathrm{O}^{6}$-benzylguanine $(\mathrm{BG})$ modified dendritic polyglycerol doxorubicin conjugates (PG-Doxo) via the disulfide bond. The anti-EGFR-PG-Doxo resulted in a 10-fold increase of uptake into EGFR-positive cancer cells compared with untargeted PG-Doxo[18]. Moreover, Schneider et al. developed VEGFR2 $\times$ Dig bispecific antibodies, which endowed the digoxigeninmodified CD31 siRNA liposome (Dig-LNP-siCD31) with VEGFR2 specificity, and anti-VEGFR2LNP-siCD3 decreased the expression of CD31 RNA about two-fold compared with LNP-siCD31 alone in MCF-7 tumors[19]. However, BG and Dig haptens are not approved for human use by the FDA, leading to a limit in the clinical application of targeted PEG-NPs. We developed the universal bispecific antibody (mPEG $\times$ HER2), which can directly modify naïve PEG-NPs and simultaneously confer HER2 targeting. Methoxy-PEG hapten has been approved by the FDA for human use to modify nanoprobes, such as SPIO, AuNP and microbubbles, to improve the biocompatibility and half-life of PEG-NPs in vivo. Our results showed that a one-step formulation of $\mathrm{mPEG} \times$ HER2 BsAbs with multiple PEG-NPs (liposome, Qdot, AuNP and SPIO) could enhance the HER2 targeting ability of PEG-NPs to HER2positive tumor cells compared with untargeted PEG-NPs. Thus, anti-mPEG BsAbs provide an easy, universal and direct formulation for any naive PEG-NPs without chemical modification which can 
accelerate the development of targeted PEG-NPs for multimodality imaging in the clinic.

Developing an easy method to produce targeted PEG-NPs against the various disease-associated markers is important for imaging. Chemical modification of antibodies on nanoparticles is currently a common method to confer targeting of a disease[20, 21]. For example, Paulis et al. coupled the antiICAM-1antibody to N-succinimidyl S-acetylthioacetate (SATA) to generate a free thiol group for conjugating with the maleimide-PEG-liposomal MRI contrast agent (L), and they proved that the binding of anti-ICAM-1 L on bEnd.5 endothelial cells could be 2-fold higher than that of L to monitor the inflammation-related ICAM-1 expression on blood vessels[22]. Chen, et al. generated anti-HER2 $\mathrm{scFv}$ containing the azide group for conjugating with the amine group of Cy5-dots to form anti-HER2 Cy5-dots, and the tumor uptake of anti-HER2 Cy5-dots had a 2.4 fold increase compared with untargeted dots in BT474 breast tumor-bearing mice[23]. However, the chemical conjugations of the antibody were random and multi-step leading to heterogeneous orientations of antibody. Furthermore, the process was time consuming[24]. Additionally, the chemical method is not universal and needs to be redesigned for different PEG-NPs. Our strategy used mPEG $\times$ HER2 to confer PEG-NPs (Lipo-DiR and SPIO) with HER2 specificity by one-step modification. Compared with untargeted nanoprobes, the cell imaging showed that the contrast intensity of $\alpha \mathrm{HER} 2 / \mathrm{Lipo}-\mathrm{DiR}$ and $\alpha \mathrm{HER} 2 / \mathrm{SPIO}$ was increased 1.6-fold and 1.75 fold on MCF7-HER2 cells, respectively. Moreover, the HER2 portion of the $\mathrm{mPEG} \times$ HER2 can be changed to other disease-associated markers such as EGFR, PSMA, CD20, TfR for development of varied $\mathrm{mPEG} \times$ marker BsAbs to easily confer nanoprobes with different specificity. Therefore, $\mathrm{mPEG} \times$ markers may provide an easy method to change the specificity of targeted nanoprobes for imaging of various diseases.

Developing a tumor-targeted contrast agent with low immunogenicity is important to allow repeat administration of the probe in humans. Protein linkers, such as streptavidin-biotin or protein A, are commonly used to provide non-covalent modification to conjugate the antibodies on nanoprobes. Jin et al. conjugated protein A on quantum dots to attach anti-CXCR4 antibody for tracking the location of CXCR4 receptors in living cells[25]. However, protein A is an exogenous protein from bacteria that may cause a risk of immunogenicity[26]. Paganelli et al. reported streptavidin as a linker to link the biotinylated $\alpha \mathrm{CEA}$ antibody and biotinylated radioactive ${ }^{111} \mathrm{In}$ tracker to increase the tumor accumulation of ${ }^{111}$ In- $\alpha$ CEA antibody in CEA-positive patients, but $63 \%$ of patients had the antistreptavidin antibody[27]. The study indicated that exogenous proteins may cause the immunogenicity in humans, limiting the application of targeted nanoprobes in the clinic[28, 29]. Here, we generated a humanized anti-mPEG BsAb (PEG $\times$ HER2) that could confer the mPEG-nanoprobe with HER2 specificity, and we demonstrated that $\alpha$ HER2/Lipo-DiR and $\alpha$ HER2/SPIO enhances accumulation in 
MCF7-HER2 tumors (HER2 ${ }^{+}$) by $161 \%$ and $186 \%$ in mice compared with MCF7/neo1 tumor (HER2-). Humanization of antibodies has been approved by FDA to reduce the immunogenicity of antibodies from non-human species, and one-half (38/78) of antibodies in clinical use are humanized[30]. Thus, the humanized anti-mPEG BsAbs may be expected to have low immunogenicity and be suitable for wide use in clinical imaging. Moreover, PEGylation of PEG-NPs could also reduce the immunogenicity of the contrast agent to prolong the half-life of contrast agents in the human body. Thus, the low immunogenicity of humanized BsAbs can confer PEG-NPs with tumor specificity by a one-step formulation and allow repeat administration of probes in humans.

\section{Conclusions}

mPEG $\times$ HER2 2 provided a simple one-step method to conjugate PEG-NPs to confer HER2specific targeting and enhanced sensitivity and contrast intensity on HER2 positive tumors for multimodality imaging. The BsAbs described here possess potential advantages for targeted imaging including: (i) anti-mPEG BsAbs could one-step modify PEG-NPs with homogeneous coupling orientations to enhance the specificity and sensitivity of imaging probes. (ii) The changeable properties and universal applicability of BsAbs can direct diverse PEG-NPs to different biomarkers expressed in various diseases for diagnosis. (iii) The humanized anti-mPEG BsAbs with low immunogenicity are suitable for direct human use. We believe that the one-step formulation of PEGylated nanoprobes with anti-mPEG BsAbs could accelerate the targeted imaging development to provide the accurate diagnoses in the clinic. 


\section{Materials and Methods}

\section{PEGylated nanoparticles}

PEGylated DOPC/CHOL Liposomes labeled with DiR (Lipo-DiR) were purchased from FormuMax Scientific (Sunnyvale, CA, USA). Superparamagnetic iron oxide (SPIO, MnMEIO- mPEG NPs) was from Prof. Yun-Ming Wang (National Chiao Tung University, Hsinchu, Taiwan). Qtracker 655 non-targeted quantum dots (Qdot) were purchased from Thermo Fisher Scientific (Waltham, USA). Gold nanoparticles (AuNP, AuNCs-PLGA-mPEG) were from Prof. Chih-Kuang Wang (Kaohsiung Medical University, Kaohsiung, Taiwan).

\section{Cells and animals}

MCF7/HER2 and MCF7/neo1 human breast adenocarcinoma cells line were grown in Dulbecco's modified Eagle's medium/nutrient mixture F-12 (DMEM/F12, Thermo Fisher Scientific, RoskildeDenmark) supplemented with 10\% (vol/vol) fetal bovine serum (FBS, Thermo, Waltham, MA, USA), $1 \%$ (vol/vol) penicillin/streptomycin (Invitrogen, Carlsbad, CA) at $37^{\circ} \mathrm{C}$ in an atmosphere of $5 \%$ (vol/vol) $\mathrm{CO}_{2}$ in air. Three to four-week-old BALB/cAnN.Cg-Foxn1nu/CrlNarl nude mice were purchased from the National Laboratory Animal Center, Taipei, Taiwan. Animal experiments were performed in accordance with institute guidelines.

\section{Bispecific antibodies and antibodies}

Human bispecific antibodies were created by linking the C-terminus of an anti-mPEG Fab (clone h15-2b[31]) to an anti-HER2 scFv or anti-DNS scFv via a flexible peptide (GGGGS)3 to form $\mathrm{mPEG} \times \mathrm{HER} 2$ and $\mathrm{mPEG} \times \mathrm{DNS}$, respectively. The anti-HER2 $\mathrm{scFv}$ was constructed by linking the 4D5 VH and VL domains with a linker (GGGGS) 3 ; the detailed description of BsAbs was as described in a previous study[17]. The VL-CK and VH-CH1-linker-scFv domains were separated with an IRES in the pLNCX retroviral vector (BD Biosciences, San Diego, CA) in the unique Hind III and Cla I restriction enzyme sites to generate $\mathrm{pLNCX}-\mathrm{mPEG} \times \mathrm{HER} 2$ and $\mathrm{pLNCX}-\mathrm{mPEG} \times \mathrm{DNS}$ plasmids. Expi293 cells were transfected with plasmids and the culture medium was collected after five days. The BsAbs were purified by affinity chromatography on gel prepared by reacting $36 \mathrm{mg}$ of O-(2Aminoethyl)-O'olyethylene glycol 750 (Sigma Aldrich) per gram of CNBr-activated sepharose 4B (GE Healthcare, Little Chalfont, UK).

\section{Bi-functional assay of mPEG $\times$ HER2 and $\mathrm{mPEG} \times \mathrm{DNS}$}


Ninety-six well plates were coated with $50 \mu \mathrm{g} / \mathrm{ml}$ of poly-D-lysine in PBS for $5 \mathrm{~min}$ at $37^{\circ} \mathrm{C}$, washed twice with deuterium depleted water and then coated with $2 \times 10^{5}$ cells/well of MCF7/HER2 $\left(\mathrm{HER}^{+}\right)$cancer cells. To fix cells, paraformaldehyde $(2 \%$, vol $/ \mathrm{vol})$ was added, left for 5 min then neutralized by $0.1 \mathrm{M}$ glycine. $\mathrm{mPEG} \times \mathrm{HER} 2$ or $\mathrm{mPEG} \times \mathrm{DNS}(10 \mu \mathrm{g} / \mathrm{ml})$ were added to the wells at room temperature for $30 \mathrm{~min}$. After extensive washing, $10 \mu \mathrm{g} / \mathrm{ml}$ of mPEG2K-BSA was added to the wells for $30 \mathrm{~min}$. After extensive washing, the bound concentrations of mPEG2K-BSA were determined by adding $10 \mu \mathrm{g} / \mathrm{ml}$ of 6-3 anti-PEG backbone antibody for $30 \mathrm{~min}$ and then adding 0.4 $\mu \mathrm{g} / \mathrm{ml}$ of goat anti-mouse IgG Fc-HRP (Jackson ImmunoResearch Laboratories). The wells were washed and then ABTS substrate was added for $30 \mathrm{~min}$ before absorbance values at $405 \mathrm{~nm}$ were measured in a microplate reader (Biochrom, St Albans, United Kingdom).

\section{One-step formulation of PEG-NPs with BsAbs}

PEG-NPs (Lipo-DiR, SPIO, Qdot and AuNP) were mixed with $\mathrm{mPEG} \times \mathrm{HER} 2$ in PBS at $4^{\circ} \mathrm{C}$ for 5 min to form $\alpha H E R 2 / P E G-N P s$, respectively. The BsAb:mPEG molar ratios of $\alpha$ HER2/PEG-NPs was 64:360 with two-fold serial dilution to 1:360. To quantify the unconjugatd BsAb in $\alpha H E R 2 / P E G-N P s$, the particles with different BsAb:mPEG ratios were incubated in mPEG2K-BSA coated 96-well plates at RT for 45 min. After extensive washing, the BsAb was detected by $0.4 \mu \mathrm{g} / \mathrm{ml}$ of goat anti-human Fab-HRP, and then ABTS substrate was added for $30 \mathrm{~min}$ before absorbance values at $405 \mathrm{~nm}$ were measured by EZ Read 400 ELISA. The BsAb-conjugation rate of $\alpha$ HER2/PEG-NPs was calculated as the total number of BsAb minus number of unconjugated BsAb, then divided by the total number of BsAb.

\section{Specificity of $\alpha H E R 2 /$ PEG-NPs for HER2 ${ }^{+}$cells}

To examine the ability of PEG-NPs (Lipo-DiR, SPIO, Qdot and AuNP) modified with various ratios of mPEG $\times$ HER2 to bind to cancer cells expressing HER2, MCF7/HER2 cells and low HER2 expression cells, MCF7/neo1 $\left(2 \times 10^{5}\right.$ cell/well) were seeded in poly-D-lysine-coated ninety-six well plates overnight at $37^{\circ} \mathrm{C}$. After fixing the cells, HER2/PLD made with the various densities of BsAb on PEG-NPs were added to the wells at RT for $20 \mathrm{~min}$. After extensive washing with PBS, the bound concentrations of PLD were determined by sequentially adding $10 \mu \mathrm{g} / \mathrm{mL}$ of $6-3$ anti-PEG antibody for $1 \mathrm{~h}$, washing with DMEM three times, and then adding $0.4 \mu \mathrm{g} / \mathrm{mL}$ of goat anti-mouse IgG Fc-HRP. The wells were washed three times with PBS and then ABTS substrate was added for 30 min before absorbance values at $405 \mathrm{~nm}$ were measured in EZ Read 400 ELISA. To further analyze HER2 specific targeting efficacy of optimized BsAb-modified PEG-NPs (Lipo-DiR, SPIO, Qdot and AuNP), serial 
dilutions of $\alpha$ HER2/PEG-NPs and $\alpha$ DNS/PEG-NPs were incubated with MCF7/HER2 cells in polyD-lysine coated 96-well plates. PLD binding was measured as described above.

\section{Fluorescence imaging of $\alpha$ HER2/Lipo-DiR in vitro and in vivo}

MCF7/HER2 $\left(5 \times 10^{6} /\right.$ well) cells were incubated with $\alpha$ HER2/Lipo-DiR, $\alpha$ DNS/Lipo-DiR or LipoDiR. After washing with PBS three times, cells were imaged with an IVIS spectrum optical imaging system (excitation, $750 \mathrm{~nm}$; emission, $780 \mathrm{~nm}$; PerkineElmer, Waltham, MA).

BALB/c nude mice bearing MCF7/HER2 (HER2 $\left.{ }^{+}\right)$and MCF7/neo1 (HER2 $\left.{ }^{-}\right)$tumors $\left(\sim 100 \mathrm{~mm}^{3}\right)$ in the mammary fat pad (m.f.p) were intravenously injected with $\alpha$ HER2/Lipo-DiR and Lipo-DiR (DiR concentration: 10 nmole per mouse), respectively. The mice were imaged on an IVIS spectrum optical imaging system at $24,48 \mathrm{~h}$ and $72 \mathrm{~h}$ after injection. The regions-of-interest (ROI) in the tumor areas were drawn and analyzed with Living Image software version 4.2 (Caliper Life Sciences).

\section{MR imaging of $\alpha \mathrm{HER2/SPIO}$ in vitro and in vivo}

MCF7/HER2 cells $\left(5 \times 10^{6}\right.$ cell/well) were incubated $\alpha$ HER2/SPIO, $\alpha$ DNS/SPIO or SPIO were added to the tubes and incubated at $37^{\circ} \mathrm{C}$ for $1 \mathrm{~h}$. After washing with PBS three times, cells precipitated at the bottom of the Eppendorf tube and were then imaged with 7T MRI (7T PharmaScan, Bruker) TR/TE, 3000/65 ms; echo train length, 10; flip angle, $150^{\circ}$; field of view, $6 \mathrm{~cm} \times 6 \mathrm{~cm}$; slice thickness, $1 \mathrm{~mm}$; interslice gap, $0.1 \mathrm{~mm}$ (10\% of slice thickness); and matrix, $192 \times 192$.

BALB/c nude mice bearing MCF7/HER2 (HER2 $\left.{ }^{+}\right)$and MCF/neo1 (HER2-) tumor $\left(\sim 100 \mathrm{~mm}^{3}\right)$ were injected intravenously with $\alpha \mathrm{HER} 2 / \mathrm{SPIO}$ and $\alpha \mathrm{DNS} / \mathrm{SPIO}(10 \mathrm{mg} / \mathrm{kg}$ per mouse) in their mammary fat pad regions, respectively. Isoflurane anesthetized mice were imaged with 7T MRI at 0 , $24 \mathrm{~h}$ after injection. TR/TE, 3000/65 ms; echo train length, 10; flip angle, 150; field of view, $4 \mathrm{~cm}$; slice thickness, $1.2 \mathrm{~mm}$; interslice gap, $0.12 \mathrm{~mm}$ (10\% of slice thickness); and matrix, $256 \times 210$. The negative enhancement was calculated by [pretreated signal intensity $\left(\mathrm{SI}_{0}\right)$-treated signal intensity $\left.\left(\mathrm{SI}_{24}\right)\right] / \mathrm{SI}_{0} * 100$. 


\section{List of abbreviations:}

PEG: polyethylene glycol

mPEG: methoxypolyethylene glycol

HER2: human epidermal growth factor receptor 2

SPIO: superparamagnetic iron oxide

Qdot: quantum dots

AuNP: gold nanoparticle

MRI: magnetic resonance imaging

EPR: enhanced permeability and retention

EGFR: human epidermal growth factor receptor

BsAb: bispecific antibody

DNS: dansyl

ELISA: enzyme-linked immunosorbent assay

Fab: antigen-binding fragment

scFv: single-chain variable fragment

HRP: horseradish peroxidase

ABTS: 2,2'-azino-bis(3-ethylbenzothiazoline-6-sulfonic acid

SD: standard deviation

ROI: region of interest

SI: signal intensity

IVIS: in vivo imaging systems

VEGFR2: vascular endothelial growth factor 2

Dig: digoxigenin

FDA: food and drug administration

ICAM-1: intercellular adhesion molecule 1

PSMA: prostate-specific membrane antigen

TfR: transferrin receptor

CXCR4: C-X-C motif chemokine receptor 4

CEA: carcinoembryonic antigen 


\section{Ethics approval and consent to participate}

Not applicable

\section{Consent for publication}

Not applicable

\section{Availability of data and materials}

All data generated or analyzed during this study are included in this published article.

\section{Competing interests}

The authors declare that they have no competing interests

\section{Funding}

This work was supported by grants from the Ministry of Science and Technology, Taipei, Taiwan (MOST 107-2320-B-037-024 -MY3, MOST 107-2320-B-037-028-MY2 and MOST 108-2311-B037-001); the National Health Research Institutes, Taiwan (NHRI-EX108-10729EI); Academia Sinica, Taiwan (AS-TP-107-L11); the Ministry of Education, Taiwan (108RSB0029); and the KMUKMUH Co-Project of Key Research (KMU-DK109001), Research Foundation (KMU-DK109004) from Kaohsiung Medical University, Taiwan. This study is also supported partially by Kaohsiung Medical University Research Center Grant (Drug Development and Value Creation Research Center) (KMU-TC108A03).

\section{Authors' contributions}

YC and TW contributed equally to this work. YC designed and performed experiments in the manuscript, analyzed data and wrote the manuscript; TW helped with experiments, data analysis and contributed to manuscript editing; YW provided the SPIO and helped experiments, data analysis for in vivo MR imaging. $\mathrm{KC}$ helped with experiments, data analysis for in vitro MR imaging. IC, YL helped with data analysis and contributed to manuscript editing; CW provided the AuNP and helped with experiments. CC helped the data analysis for in vivo experiments. RL, CJ, TL, EL helped with experiments and data analysis. TC provided concept, technical guidance with bispecific antibody design and contributed to manuscript editing. FC provided the information of breast cancer clinical treatments and current challenges and contributed to manuscript writing and editing. All authors read and approved the final manuscript. 
Acknowledgements

Not applicable 


\section{Reference}

1. Napp J, Mathejczyk JE, Alves F: Optical imaging in vivo with a focus on paediatric disease: technical progress, current preclinical and clinical applications and future perspectives. Pediatric radiology 2011, 41(2):161-175.

2. Catana C, Guimaraes AR, Rosen BR: PET and MR imaging: the odd couple or a match made in heaven? Journal of nuclear medicine : official publication, Society of Nuclear Medicine 2013, 54(5):815-824.

3. Madsen MT, Park $\mathrm{CH}$ : Enhancement of SPECT images by Fourier filtering the projection image set. Journal of nuclear medicine : official publication, Society of Nuclear Medicine 1985, 26(4):395-402.

4. Bobo D, Robinson KJ, Islam J, Thurecht KJ, Corrie SR: Nanoparticle-Based Medicines: A Review of FDA-Approved Materials and Clinical Trials to Date. Pharmaceutical research 2016, 33(10):2373-2387.

5. Jokerst JV, Lobovkina T, Zare RN, Gambhir SS: Nanoparticle PEGylation for imaging and therapy. Nanomedicine 2011, 6(4):715-728.

6. Kirpotin DB, Drummond DC, Shao Y, Shalaby MR, Hong K, Nielsen UB, Marks JD, Benz CC, Park JW: Antibody targeting of long-circulating lipidic nanoparticles does not increase tumor localization but does increase internalization in animal models. Cancer research 2006, 66(13):6732-6740.

7. Chen L, Zang F, Wu H, Li J, Xie J, Ma M, Gu N, Zhang Y: Using PEGylated magnetic nanoparticles to describe the EPR effect in tumor for predicting therapeutic efficacy of micelle drugs. Nanoscale 2018, 10(4):1788-1797.

8. Freedman M, Chang EH, Zhou Q, Pirollo KF: Nanodelivery of MRI contrast agent enhances sensitivity of detection of lung cancer metastases. Academic radiology 2009, 16(5):627-637.

9. Cheng CM, Chu PY, Chuang KH, Roffler SR, Kao CH, Tseng WL, Shiea J, Chang WD, Su YC, Chen $\mathrm{BM}$ et al: Hapten-derivatized nanoparticle targeting and imaging of gene expression by multimodality imaging systems. Cancer gene therapy 2009, 16(1):83-90.

10. Lozano N, Al-Ahmady ZS, Beziere NS, Ntziachristos V, Kostarelos K: Monoclonal antibodytargeted PEGylated liposome-ICG encapsulating doxorubicin as a potential theranostic agent. International journal of pharmaceutics 2015, 482(1-2):2-10.

11. Zhou Z, Zhang C, Qian Q, Ma J, Huang P, Zhang X, Pan L, Gao G, Fu H, Fu S et al: Folic acidconjugated silica capped gold nanoclusters for targeted fluorescence/X-ray computed tomography imaging. J Nanobiotechnology 2013, 11:17.

12. Zhu L, Guo Y, Wang L, Fan X, Xiong X, Fang K, Xu D: Construction of ultrasonic nanobubbles carrying CAIX polypeptides to target carcinoma cells derived from various organs. J Nanobiotechnology 2017, 15(1):63.

13. Freedman M, Chang EH, Zhou Q, Pirollo KF: Nanodelivery of MRI contrast agent enhances sensitivity of detection of lung cancer metastases. Academic radiology 2009, 16(5):627-637. 
14. Wu SC, Chen YJ, Wang HC, Chou MY, Chang TY, Yuan SS, Chen CY, Hou MF, Hsu JT, Wang YM: Bispecific Antibody Conjugated Manganese-Based Magnetic Engineered Iron Oxide for Imaging of HER2/neu- and EGFR-Expressing Tumors. Theranostics 2016, 6(1):118-130.

15. Hajdu P, Chimote AA, Thompson TH, Koo Y, Yun Y, Conforti L: Functionalized liposomes loaded with siRNAs targeting ion channels in effector memory $T$ cells as a potential therapy for autoimmunity. Biomaterials 2013, 34(38):10249-10257.

16. Sun X, Zhang G, Patel D, Stephens D, Gobin AM: Targeted cancer therapy by immunoconjugated gold-gold sulfide nanoparticles using Protein $\mathbf{G}$ as a cofactor. Annals of biomedical engineering 2012, 40(10):2131-2139.

17. Cheng YA, Chen IJ, Su YC, Cheng KW, Lu YC, Lin WW, Hsieh YC, Kao CH, Chen FM, Roffler SR et al: Enhanced drug internalization and therapeutic efficacy of PEGylated nanoparticles by one-step formulation with anti-mPEG bispecific antibody in intrinsic drug-resistant breast cancer. Biomaterials science 2019, 7(8):3404-3417.

18. Hussain AF, Kruger HR, Kampmeier F, Weissbach T, Licha K, Kratz F, Haag R, Calderon M, Barth S: Targeted delivery of dendritic polyglycerol-doxorubicin conjugates by scFv-SNAP fusion protein suppresses EGFR+ cancer cell growth. Biomacromolecules 2013, 14(8):25102520.

19. Schneider B, Grote M, John M, Haas A, Bramlage B, Ickenstein LM, Jahn-Hofmann K, Bauss F, Cheng W, Croasdale R et al: Targeted siRNA Delivery and mRNA Knockdown Mediated by Bispecific Digoxigenin-binding Antibodies. Molecular therapy Nucleic acids 2012, 1:e46.

20. Li S, Chen L, Wang G, Xu L, Hou S, Chen Z, Xu X, Wang X, Liu F, Du YZ: Anti-ICAM-1 antibodymodified nanostructured lipid carriers: a pulmonary vascular endothelium-targeted device for acute lung injury therapy. J Nanobiotechnology 2018, 16(1):105.

21. Han $Y, A n Y$, Jia $G$, Wang $X$, He $C$, Ding $Y$, Tang $Q$ : Facile assembly of upconversion nanoparticle-based micelles for active targeted dual-mode imaging in pancreatic cancer. $J$ Nanobiotechnology 2018, 16(1):7.

22. Paulis LE, Jacobs I, van den Akker NM, Geelen T, Molin DG, Starmans LW, Nicolay K, Strijkers GJ: Targeting of ICAM-1 on vascular endothelium under static and shear stress conditions using a liposomal Gd-based MRI contrast agent. Journal of nanobiotechnology 2012, 10(1):25.

23. Chen F, Ma K, Madajewski B, Zhuang L, Zhang L, Rickert K, Marelli M, Yoo B, Turker MZ, Overholtzer $M$ et al: Ultrasmall targeted nanoparticles with engineered antibody fragments for imaging detection of HER2-overexpressing breast cancer. Nature communications 2018, 9(1):4141.

24. Cheng Z, Al Zaki A, Hui JZ, Muzykantov VR, Tsourkas A: Multifunctional nanoparticles: cost versus benefit of adding targeting and imaging capabilities. Science 2012, 338(6109):903910.

25. Jin T, Tiwari DK, Tanaka S-i, Inouye Y, Yoshizawa K, Watanabe TM: Antibody-ProteinA 
conjugated quantum dots for multiplexed imaging of surface receptors in living cells. Molecular BioSystems 2010, 6(11):2325-2331.

26. Leonetti M, Thai R, Cotton J, Leroy S, Drevet P, Ducancel F, Boulain JC, Menez A: Increasing immunogenicity of antigens fused to Ig-binding proteins by cell surface targeting. Journal of immunology 1998, 160(8):3820-3827.

27. Paganelli G, Chinol M, Maggiolo M, Sidoli A, Corti A, Baroni S, Siccardi AG: The three-step pretargeting approach reduces the human anti-mouse antibody response in patients submitted to radioimmunoscintigraphy and radioimmunotherapy. European journal of nuclear medicine 1997, 24(3):350-351.

28. Narain R: Chemistry of bioconjugates: synthesis, characterization, and biomedical applications: John Wiley \& Sons; 2013.

29. Hock MB, Thudium KE, Carrasco-Triguero M, Schwabe NF: Immunogenicity of antibody drug conjugates: bioanalytical methods and monitoring strategy for a novel therapeutic modality. The AAPS journal 2015, 17(1):35-43.

30. Scott AM, Wolchok JD, Old LJ: Antibody therapy of cancer. Nature reviews cancer 2012, 12(4):278-287.

31. Kao CH, Wang JY, Chuang KH, Chuang CH, Cheng TC, Hsieh YC, Tseng YL, Chen BM, Roffler SR, Cheng TL: One-step mixing with humanized anti-mPEG bispecific antibody enhances tumor accumulation and therapeutic efficacy of mPEGylated nanoparticles. Biomaterials 2014, 35(37):9930-9940. 


\section{Figures}

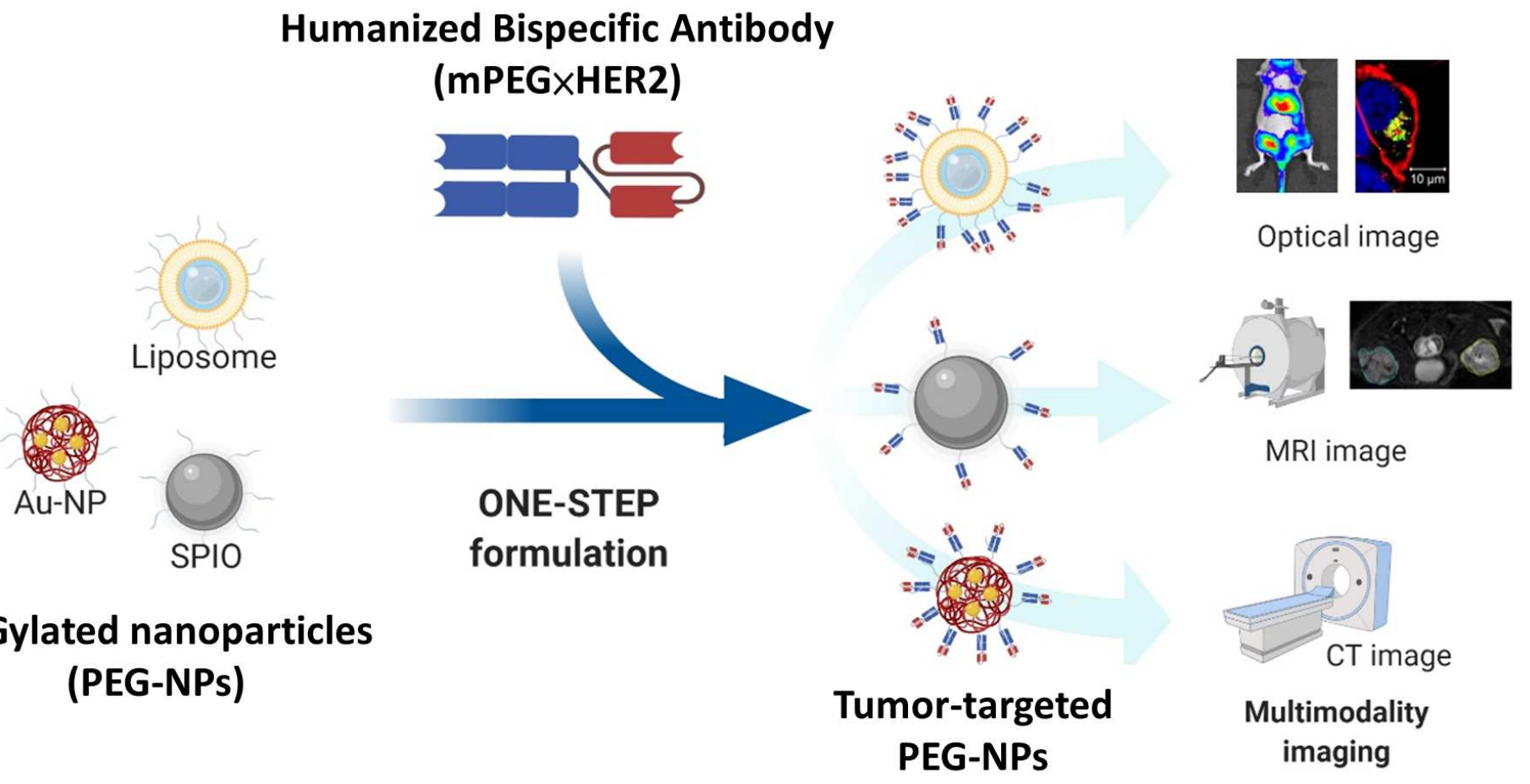

Figure 1

Humanized Bispecific Antibody (mPEGxHER2) Rapidly Confers PEG-NPs Tumor Specificity for Multimodality Imaging in Breast Cancer anti-mPEG BsAbs (mPEG×markers) provide an easy, universal and one-step formulation for any naive PEG-NPs to accelerate the development of targeted PEG-NPs for multimodality imaging in the clinic. 
(A)

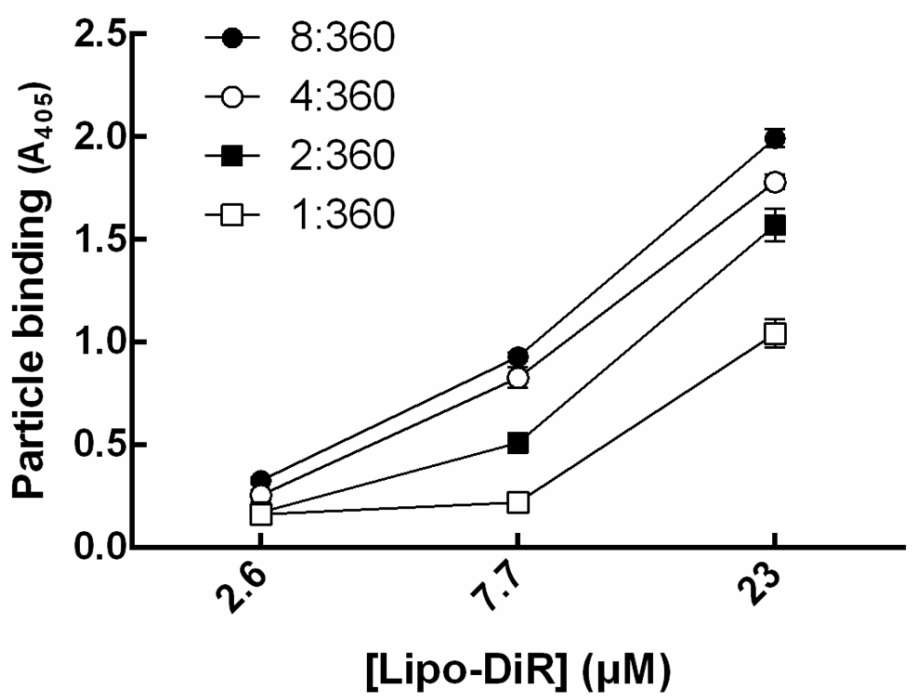

(C)

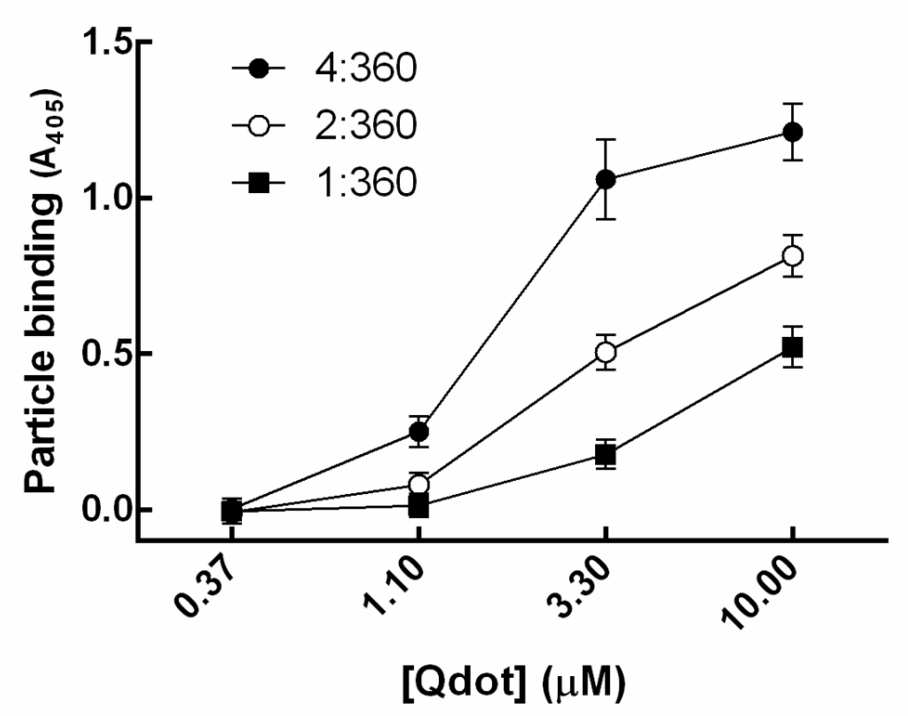

(B)

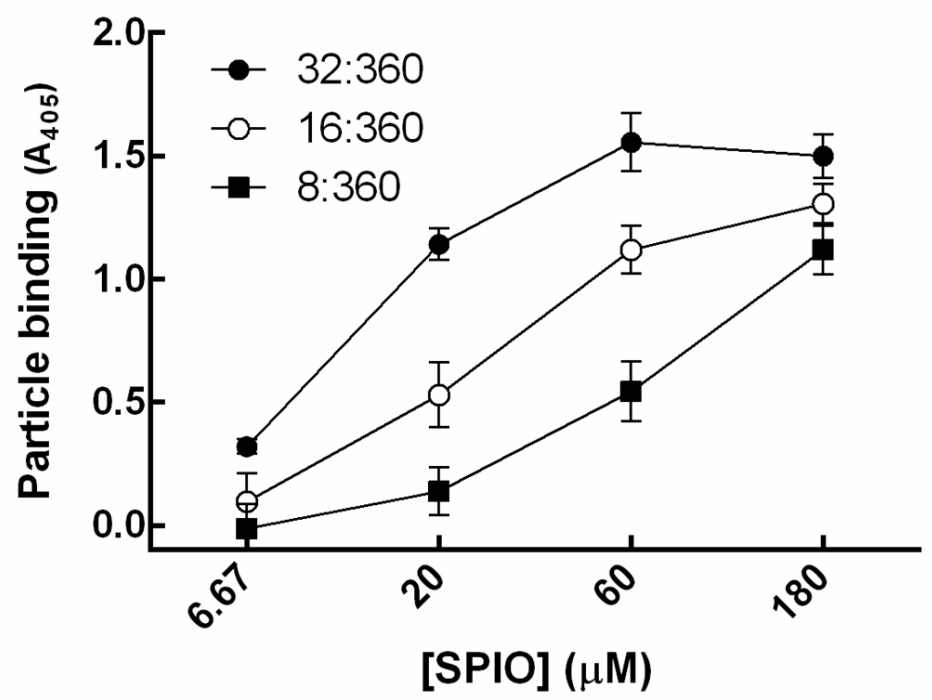

(D)

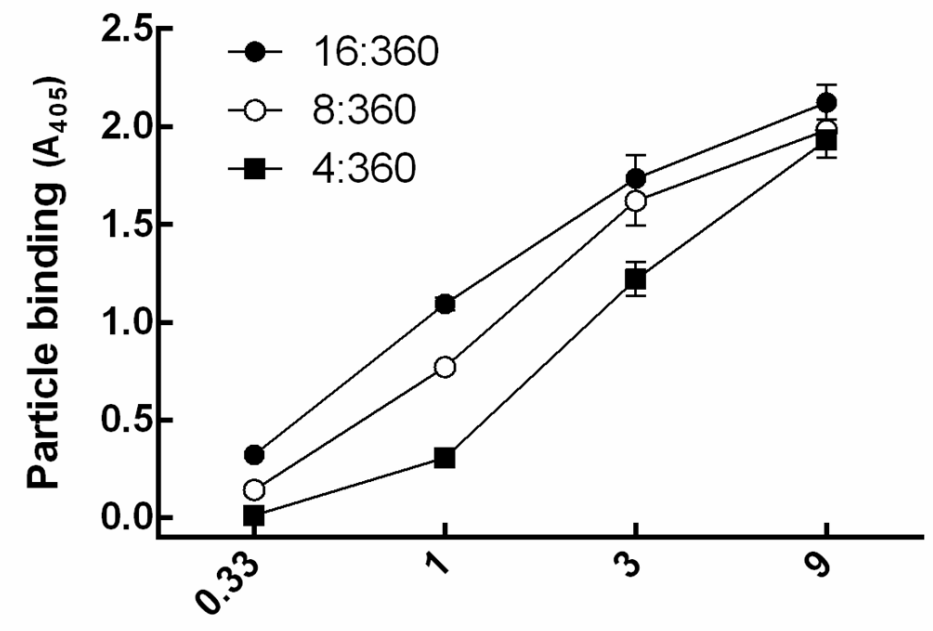

$[$ AuNP] $(\mu \mathrm{g} / \mathrm{ml})$

Figure 2

The HER2 binding ability of aHER2/PEG-NPs Different BsAb:mPEG ratios of (A) aHER2/Lipo-DiR, (B) aHER2/SPIO, (C) aHER2/Qdot and (D) aHER2/AuNP were incubated with MCF7/HER2, and then anti-PEG antibody was added to detect PEG-NPs via ELISA ( $n=3$, triplicate). Bars, SD. 
(A)

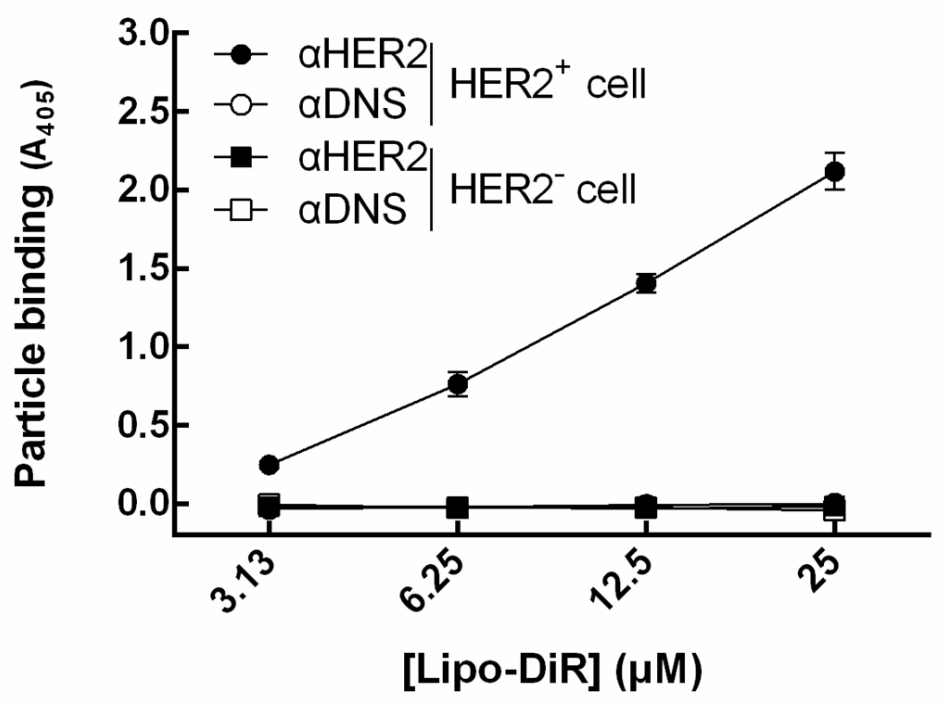

(C)

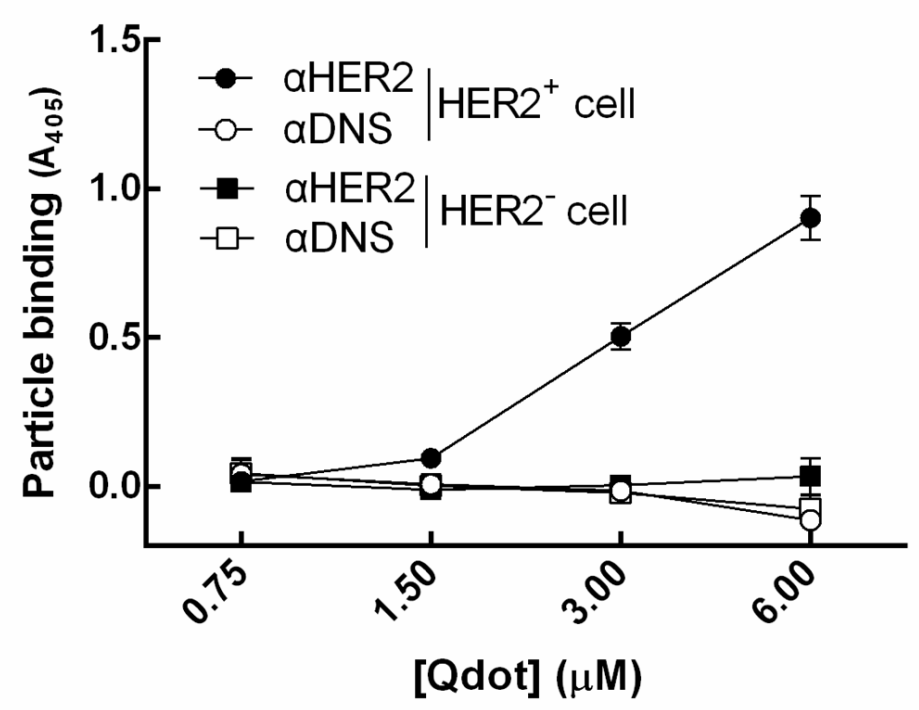

(B)

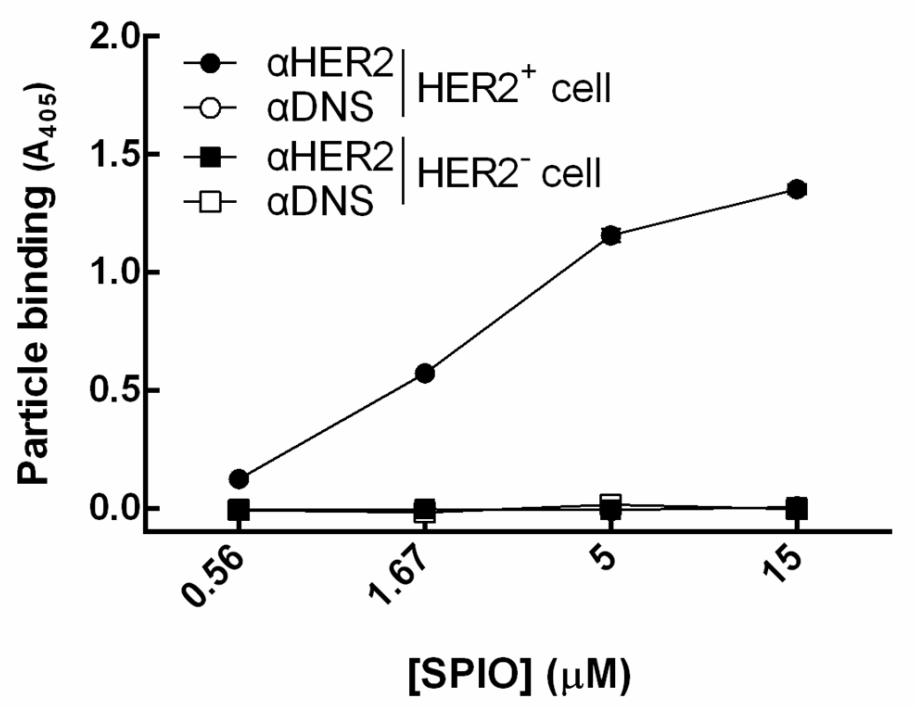

(D)

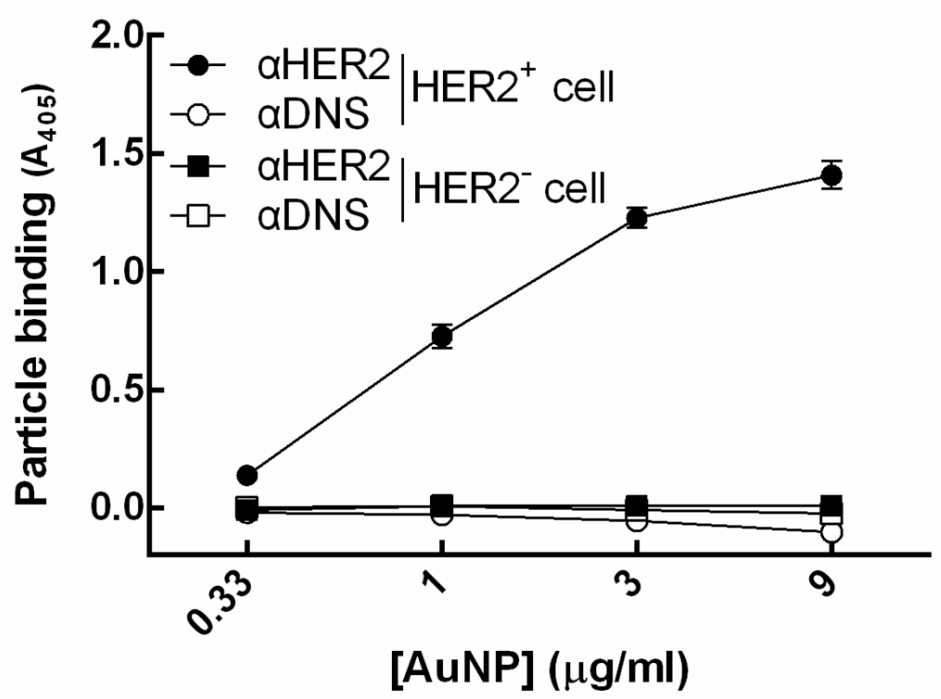

Figure 3

Specificity of aHER2/PEG-NPs for HER2+ cancer cells MCF7/HER2 (HER+) (circle shape) and MCF7/neo1(HER2-) (square shape) cancer cells in 96-well plates were incubated with mPEG×HER2 (solid shape) and mPEG $\times$ DNS (hollow shape) modified with different contrast agents. After washing, bound contrast agents were detected by ELISA ( $n=3$, triplicate). Bars, SD. 
(A) Lipo-DiR $\begin{gathered}\alpha D N S / \\ \text { Lipo-DiR Lipo-DiR }\end{gathered}$

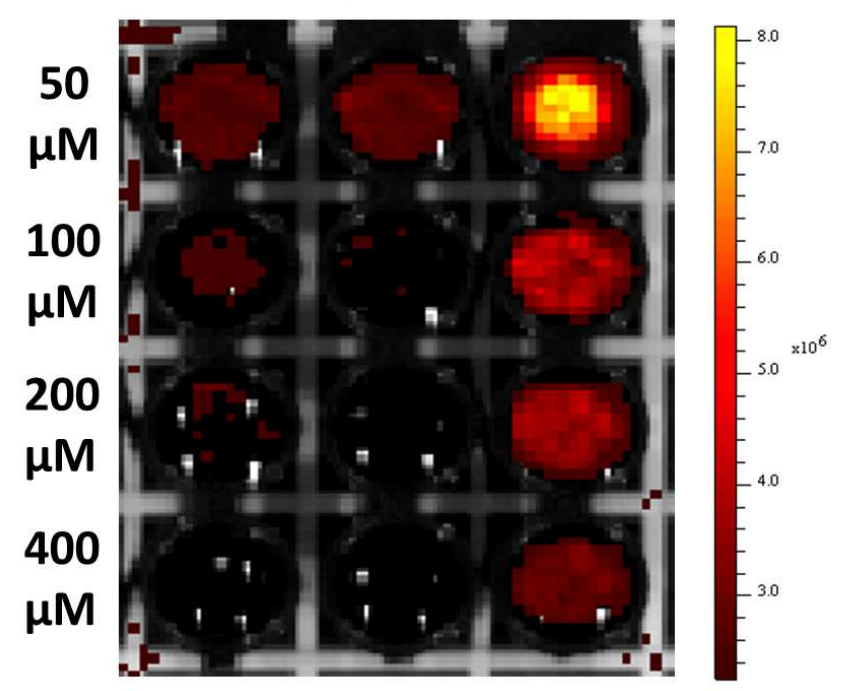

(C)

$$
\text { SPIO /SPIO /SPIO }
$$

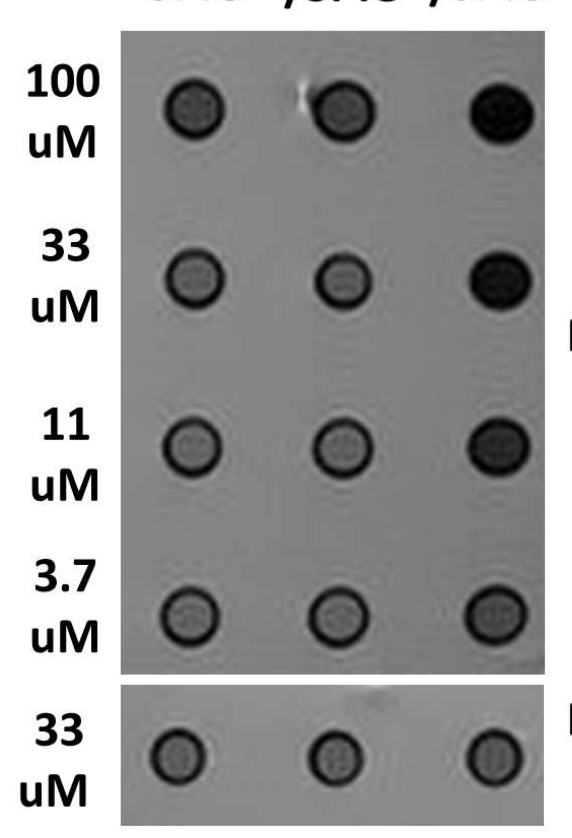

(B)

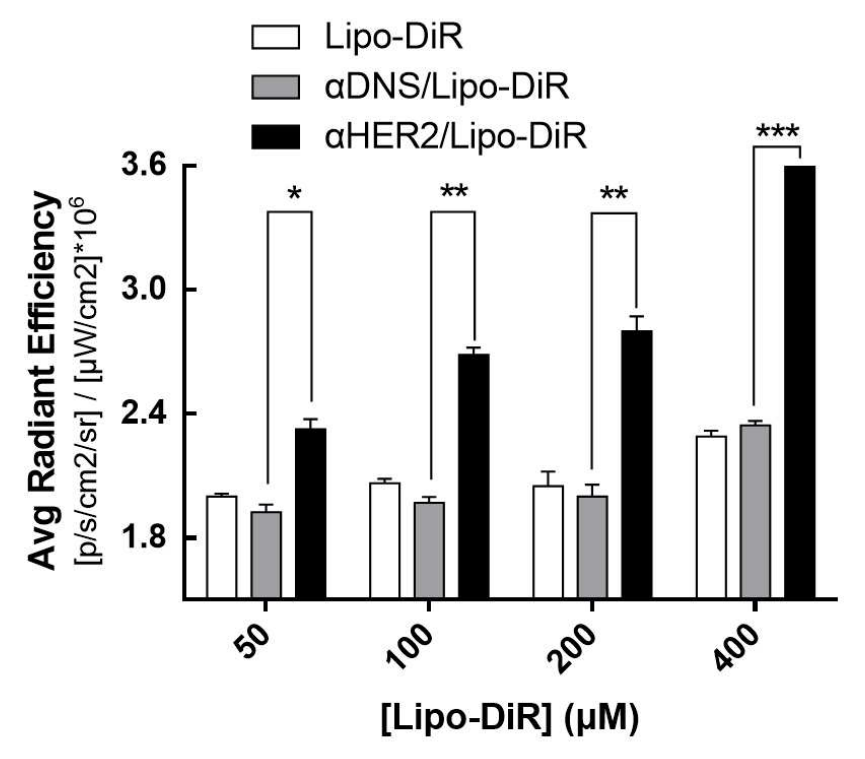

(D)

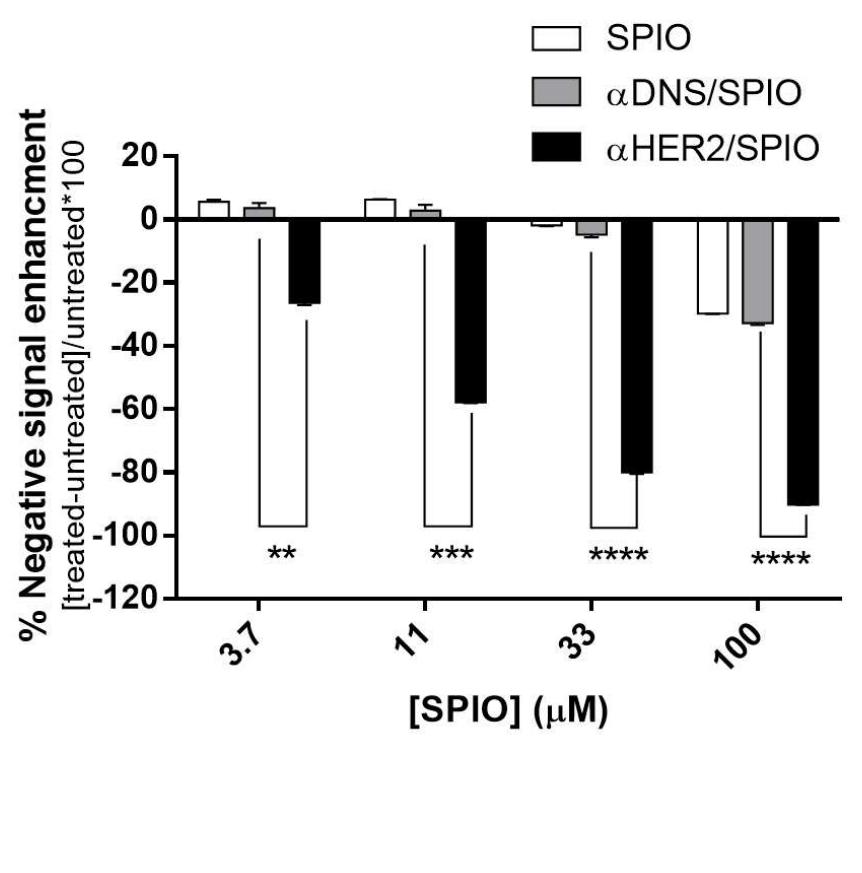

Figure 4

In vitro sensitivity image of aHER2/PEG-NPs MCF7/HER2 (HER+) cancer cells incubated with HER2 targeted-contrast agent serial dilution concentration. (A) aHER2/Lipo-DiR, aDNS/Lipo-DiR and Lipo-DiR was added to cells. Fluorescence images were obtained by the IVIS spectrum system. (B) The result was the calculation of average radiant efficiency of (A). (C) aHER2/SPIO, aDNS/SPIO and SPIO was added to cells. MR imaging was performed with a 7.0 T MR imaging scanner. (D) The result from (C) was

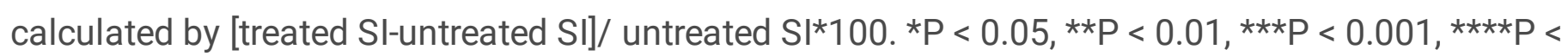
0.0001 (unpaired $t$ test). 
(A)

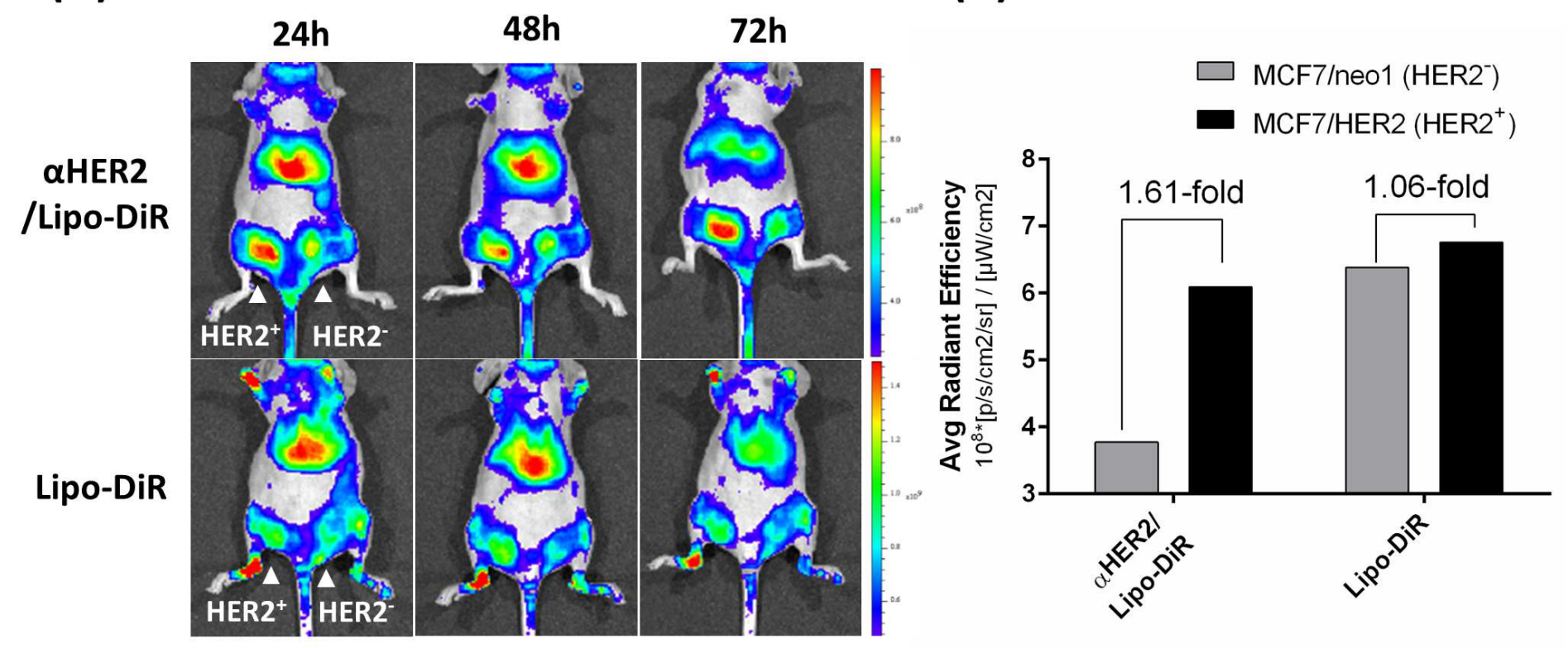

Figure 5

In vivo IVIS imaging of aHER2/SPIO and aDNS/SPIO The tumor delivery of aHER2/PLD in HER2overexpressing tumors. (A) aHER2/Lipo-DiR and Lipo- DiR were intravenously injected in mice bearing HER2high (left flank) and HER2low (right flank) tumors. The fluorescence intensity of DiR was detected at $24 \mathrm{~h}$ and $72 \mathrm{~h}$ after injection by IVIS. (B) Quantification of average radiant efficiency in HER2+ tumor and HER2- tumor at $72 \mathrm{~h}$. 
(A)

$\alpha$ HER2/ SPIO

$\mathrm{Ohr}$

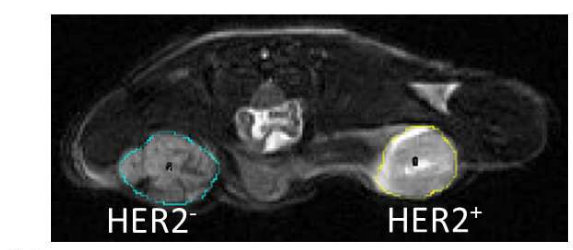

Mean 100.2

SI

aDNS/SPIO

$\mathrm{Ohr}$

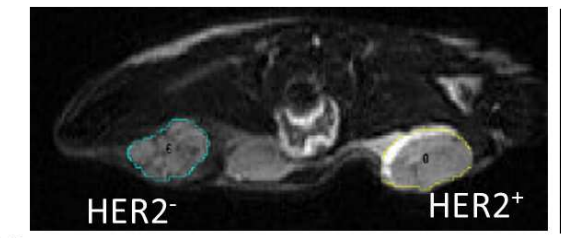

Mean 95.3 SI
166.0

142.9
aHER2/ SPIO

$24 \mathrm{hr}$

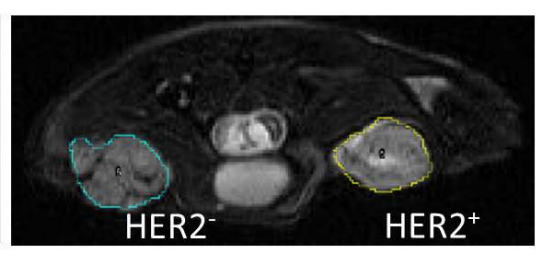

82.7 $\alpha D N S / S P I O$ 24hr

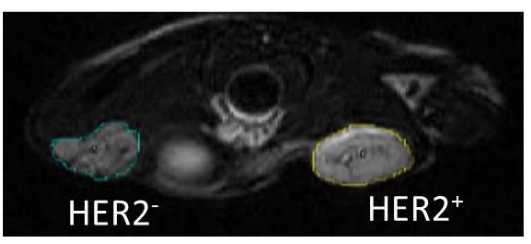

73.0
(B)

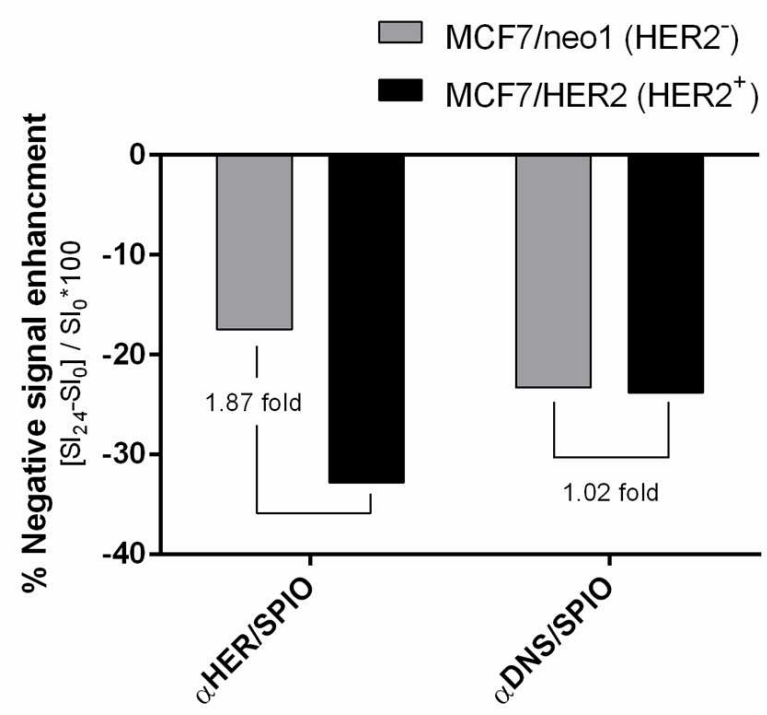

Figure 6

In vivo MR imaging of aHER2/SPIO and aDNS/SPIO Nude mice bearing MCF7/HER2 (right m.f.p) and MCF7/neo1 (left m.f.p) tumors were intravenously injected with aHER2/SPIO and aDNS/ SPIO (10 $\mathrm{mg} / \mathrm{kg}$ ). (A) Mice were sequentially imaged at pre- treatment and $24 \mathrm{~h}$ with a $7.0 \mathrm{~T}$ MR imaging scanner. (B) The result was calculated as the percentage of negative signal enhancement by [pretreated signal intensity (SI0)-treated signal intensity (SI24)]/SIO*100.

\section{Supplementary Files}

This is a list of supplementary files associated with this preprint. Click to download.

- ST11.tif 